\title{
Ricerche sui sistemi lineari di curve algebriche di genere qualunque. $\left.{ }^{*}\right)$
}

\author{
(Memoria del prof. G. JENG, a Milano.)
}

Il problema di cui mi occupo in questa Memoria si è quello di assegnare i sistemi lineari generali d'ordine minimo di un dato genere $p$ e di un dato grado $k$ o per lo meno d'indicare criterii generali per determinarli ( $\$ \$ 5-7$ ); problema che, limitato ai sistemi triplamente infiniti e alle reti, si riattacca con altre questioni relative alla rappresentazione delle superficie e alla teorica delle trasformazioni piane multiple. Aggruppati i sistemi lineari generali in famiglie (\$2), distinti quelli di minimo ordine in normali e derivati e classificatili in tipi monomi, binomi e trinomi ( $\$ 4)$, trovo una relazione invariantiva fra il numero dei punti fondamentali e il numero delle linee fondamentali, tanto pei sistemi lineari generali $(\S 3)$, quanto pei sistemi speciali $(\S 9)$, che sono fra loro riducibili mediante trasformazioni quadratiche. Indico all' ultimo $(\$ 10-11)$ i sistemi di minimo ordine relativi ai generi $p=0,1,2,3,4$, insieme a parecchi teoremi che vi si riferiscono $(\$ 8)$; non senza mettere in rilievo (n. ${ }^{\circ} 67$ ) le questioni accessorie che l'esposto metodo lascia insolute.

\section{$\S 1$. Generalità sui sistemi lineari a punti fondamentali arbitrarii.}

1. In un piano sono dati in posizioni distinte affatto arbitrarie e fra loro indipendenti $f$ punti fondamentali $a_{1}, a_{2}, \ldots a_{f}$ coi rispettivi gradi di moltiplicità $r_{1}, r_{2}, \ldots r_{f}\left(\alpha_{i}\right.$ punto fondamentale $r_{i}$-plo) che si suppongono disposti

(*) Mentre questa Memoria aspettava il turno dinserzione negli Annali ne comunicaj al R. Istituto Lombardo alcune parti, riassunte nei Rendiconti dell' Istituto (ser. $2^{2}, \mathrm{t} . \mathrm{XX}$, adunanza 31 marzo 1887); tale riassunto avrekbe richiesto una Errata-corrige, che però ho stimato inutile di pubblicare.

Annali di Matematica, tomo XV. 
in ordine di grandezza decrescente, cosicchè $r_{1} \equiv r_{2} \equiv \cdots \equiv r_{f}$. L'ipotesi che i punti $a_{i}$ abbiano posizioni speciali sarà considerata a parte $(\S 9)$.

La totalità $\infty^{c^{\prime}}$ di curve algebriche dell'ordine $M$ e del genere $p$, non spezzantisi in parti e passanti con $r_{i}$ rami pei punti $a_{i}(i=1,2, \ldots f)-$ ma non vincolate da nessun'altra condizione comune all'infuori di quelle o direttamente assorbite dai passaggi pei punti fondamentali o indirettamente ma univocamente determinate da tali passaggi - costituisce un sistema lineare generale $\left(C_{M}\right)$ del genere $p$ e dell'ordine $M$. Chiamo dimensione del sistema il numero $c^{i}$; e chiamo grado il numero $k$ delle intersezioni, esterne ai punti $\alpha_{i}$, di due $C_{M}$ qualunque.

Se fra queste intersezioni ve ne sono di fisse, cioè comuni a tutte quante le curve di $\left(C_{M}\right)$, esse completano coi punti fondamentali la base del sistema (cfr. n. $\left.{ }^{\mathrm{i}} 54,57\right)$. Per esempio, quando il sistema è un fascio $\left(c^{\prime}=1\right)$, le intersezioni di due $C_{A T}$, esterne ai punti $a_{i}$, sono tutte fisse e il grado $k$ esprime esclusivamente il numero dei punti base che sono determinati dai punti fondamentali.

2. Il sistema lineare poc'anzi definito è completamente determinato dai punti fondamentali arbitrarii $a_{i}$, perchè senza eccezione ogni curva di ordine $M$ e di genere $p$, avente un punto $r_{i}$-plo in $a_{i}(i=1,2, \ldots f)$, fa parte del sistema; onde si avrà:

dalle quali si ricava:

$$
\begin{aligned}
\frac{M(M+3)}{2} & =\frac{\Sigma r(r+1)}{2}+c^{\prime} \\
\frac{(M-1)(M-2)}{2} & =\frac{\Sigma r(r-1)}{2}+\jmath \\
M^{2} & =\Sigma r^{2}+k,
\end{aligned}
$$

$$
c^{\prime}=k+1-p
$$

equazione di condizione che collega le tre caratteristiche $p, k, c^{\prime}$ di un sistema lineare generale e determinato (dai punti fondamentali arbitrarii). Se $c^{\prime}=1$ si ha: in un fascio generale il grado $k$ è uguale al genere $p$.

3. In un sistema $S$, generale e determinato, sono contenuti $c^{\prime}-1$ altri sistemi lineari, aventi gli stessi punti fondamentali arbitrarii $a_{i}$ e che sono di ugual genere, di ugual grado e di ugual ordine del dato, ma di dimensione minore; $c^{\prime \prime}+1$ curve arbitrarie $C_{M}$ di $S$ non appartenenti a un medesimo sistema di dimensione $<c^{\prime \prime}$, e non aventi altri punti arbitrarii comuni oltre agli 
$a_{i}$, staccano dal sistema dato un sistema minore $\boldsymbol{\infty}^{r^{\prime \prime \prime}}$ o di dimensione $c^{\prime \prime}$. Questi sistemi minori, contenuti nel sistema dato, sono incompletamente determinati dai loro punti fondamentali arbitrarii; fra le loro caratteristiche $p, k, e^{\prime \prime}$ ha luggo la relazione:

$$
r<+1-p
$$

4. A raphesentare un sistema lineare s del genere $\mu$, del grado li c della dimensione $c^{\prime}$ di curve piane d'ordine $M$, useremo secondo i casi uno dei simboli equivalenti:

$$
S \equiv\left(C_{M}\right)_{p, c^{\prime}}^{k} \equiv\left(C_{H J}\right)_{p}^{k_{p}} \equiv\left(C_{J I}\right)_{p, c^{\prime}} \equiv\left(C_{M}\right)_{p^{\prime}} \equiv\left(C_{H I} \equiv\left(p, k, c^{\prime}\right)_{H},\right.
$$

nei quali sono messe in evidenza le sue caratteristiche; oppure uno dei simboli:

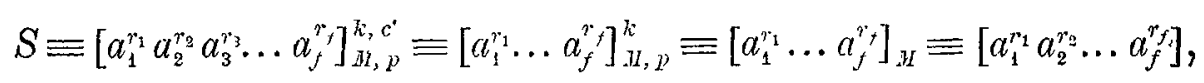

nei quali sono messi in evidenza anche $i$ suoi punti fondamentali.

5. Indicheremo con $f_{c}$ il numero delle linee fondamentali del sistema: cioè delle curve d'ordine $<M$ dotate della proprietà di non essere incontrate da una $C_{3 I}$ qualunque esternamente ai punti fondamentali $a_{1}, a_{2}, \ldots a_{f}$.

\section{$\S 2$. Famiglie di sistemi lineari. - Sistemi di minimo ordine.}

6. Un sistema lineare di qualsivoglia genere e grado appartiene a una famiglia di sistemi lineari (di ugual genere e ugual dimensione, ma di ordine in generale differente), i quali provengono dal dato mediante una o più trasformazioni quadratiche successive. Fra i sistemi di una stessa famiglia ve n' ha uno (sistema tipico, sistema minimo o di ordine minimo) il cui ordine $\mu$ non può ulteriormente essere abbassato da qualsiasi trasformazione quadratica; esso è caratterizzato dalla condizione:

$$
r_{1}+r_{2}+r_{3}=\mu \text {. }
$$

Infatti se la (5) non è verificata, collocando i punti fondamentali di una trasformazione quadratica $T_{2}$ nei primi tre $\left(\mathrm{n}^{\circ}{ }^{\circ} 1\right)$ punti fondamentali del sistema dato, questo si trasforma in altro di ordine minore.

Se al contrario la condizione (5) è sodisfatta, comunque si assumano i punti fondamentali della trasformazione quadratica, il sistema dato $\left(C_{\mu}\right)$ si trasforma in altro di ordine non minore del numero $2 \mu-\left(r_{1}+r_{2}+r_{3}\right)$; il quale nu-

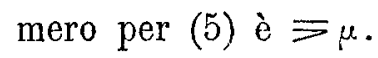


7. Quando $r_{1}+r_{2}+r_{3}=\mu$ il sistema generale d'ordine minimo

$$
\left(C_{\mu}\right) \equiv\left[a^{r_{1}} b^{r_{3}} e^{r_{3}} \ldots l_{i}^{r_{i}} \ldots l_{f}^{f_{f}}\right]
$$

¿̀ trasformabile in sè stesso. Infatti mediante la trasformazione quadratica $T_{2} \equiv(a b c, \alpha \beta \gamma)$ le curve del sistema trasformato sono appunto dell'ordine $2 \mu-\left(r_{1}+r_{2}+r_{3}\right)=\mu$; le indicheremo con $C_{\mu}^{\prime}$. Quanto ai punti $\alpha, \beta, \gamma$ essi sono rispettivamente multipli per le $C^{\prime}{ }_{\mu}$ secondo i numeri

$$
\mu-\left(r_{2}+r_{3}\right)=r_{1}, \quad \mu-\left(r_{3}+r_{1}\right)=r_{2}, \quad \mu-\left(r_{1}+r_{2}\right)=r_{3},
$$

e ogni punto $r_{i}$-plo $l_{i}(i \equiv 4)$ delle $C_{\mu}$ dà luogo a un punto equimolteplice $\lambda_{i}$ per le $C_{\mu}^{\prime}{ }_{\mu}\left(\lambda_{i}\right.$ trasformato quadraticamente di $\left.l_{i}\right)$; cosicchè $\left(C_{\mu}\right)$ si cambia, mediante la $T_{2}$, nel sistema $\left(C_{\mu}^{\prime}\right) \equiv\left[\alpha^{r_{1}^{\prime}} \beta^{r_{2}} \gamma^{{ }^{\prime}} \ldots \lambda_{i}^{r_{1}} \ldots \lambda_{f}^{r_{f}}\right]$ che ha genere, grado e dimensione uguali a quelli del dato, perchè l'indicata trasformazione quadratica lascia invariati questi elementi. Inoltre $\left(C_{\mu}\right)$ e $\left(C^{\prime}{ }_{\mu}\right)$ hanno lo stesso numero di punti fondamentali arbitrarii e questi sono rispettivamente affetti dagli stessi gradi di moltiplicità; onde i due sistemi generali si possono riguardare come identici; in altri termini mediante l'indicata $T_{2}$ il sistema $\left(C_{\mu}\right)$ si trasforma in sè stesso. Ogni altra $T_{2}$ condurrebbe a un sistema trasformato di ordine $>\mu$.

8. Due differenti sistemi generali d'ordine minimo, anche se di ugual genere, ugual grado e ugual dimensione, sono fra loro irriducibili. Infatti siano $\left(C_{\mu^{\prime}}\right)$ e $\left(C_{\mu}\right)$ i due sistemi d'ordine minimo, di genere $p$, di grado $k$ e di diinensione $c^{\prime}$ :

$1 .^{n}$ se $\mu^{\prime}=\mu$, i due sistemi, pel numero precedente, o sono irriducibili o contro l'ipotesi si possono riguardare come identici;

$2 .^{\circ}$ se $\mu^{\prime}>\mu$, il primo sistema, essendo d'ordine minimo, non potrà trasformarsi nell'altro, ch'è d'ordine minore.

9. A un dato genere $p$ e a un dato grado $k$ corrispondono tante famiglie distinte di sistemi lineari, quanti sono $i$ relativi sistemi d'ordine minimo.

10. Se un sistema generale del minimo ordine possiede due soli punti fondamentali $a, b$, multipli secondo $r_{1}, r_{2}$ e se $r_{1}+r_{2}=M$, la retta $a b$, non essendo incontrata in punti variabili da una $C_{M I}$ qualunque, è manifestamente una linea fondamentale; d'altra parte, come al $\mathrm{n}^{0} 7$ si dimostra che il sistema è bensi trasformabile in sè stesso, ma non può ridursi ad altro di ordine minore, cosicchè esso è di ordine minimo. 
Escluso questo caso, si riconosce [imitando la dimostrazione di Caporall $\left({ }^{*}\right)$ ] che in ogni altro sistema lineare dotato di punti fondamentali arbitrarii e di almeno una curva fondamentale, la somma dei tre gradi più elevati di moltiplicità supera l'ordine del sistema; quindi se $r_{1}+r_{2}+r_{3}=M$, cioè se il sistema (generale) è di ordine minimo, e si esclude il caso eccezionale testè considerato, esso non può contenere curve fondamentali. Si ha dunque il teorema:

I. - Un sistema generale del minimo ordine, di qualsivoglia genere e grado, è privo di linee fondamentali; eccettuato il caso che esso possieda due soli punti fondamentali, la somma dei cui gradi di moltiplicità uguagli l'ordine del sistema.

\section{$\S 3$. Proprietà invariantiva dei sistemi appartenenti a una stessa famiglia. Eccesso degli elementi fondamentali.}

\section{Sia}

$$
\left(C_{\mu}\right) \equiv\left[a_{1}^{r_{1}} a_{2}^{\gamma^{2}} a_{3}^{\gamma_{3}} \ldots a_{f_{0}}^{r_{f_{0}}}\right]
$$

il sistema di minimo ordine corrispondente a una data famiglia $\left(p k c^{\prime} \mu\right)$ di sistemi lineari generali, e si supponga che il gruppo $(k)$ espresso dal grado consti esclusivamente di punti variabili $\left(^{* *}\right)$. Ogni sistema appartenente alla medesima potrà evjdentemente ricavarsi da $\left(C_{\mu}\right)$ mediante un certo numero di trasformazioni quadratiche successive o ch' è lo stesso mediante una opportuna trasformazione Cremoniana $T_{n}$ di ordine $n$.

12. Poniamo

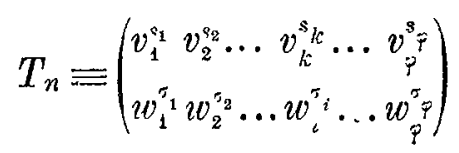

ove i simboli $v_{1}, v_{2}, \ldots v_{\varphi}$ indicano i $\varphi$ punti fondamentali $s_{k}$-pli del primo piano (quello del sistema dato); e i simboli $w_{1}, w_{2}, \ldots w_{\varphi}$ i $\varphi$ punti fondamentali $\sigma_{i}$-pli dell'altro piano (quello del sistema trasformato, che si tratta di determinare);

$\left.{ }^{*}\right)$ Sopra $i$ sistemi lineari triplamente infiniti, ecc., n. ${ }^{\mathrm{i}}$, 3. (Collectanea Mathematica, Milano, 1881.)

(*) Per sistemi generali nei quali non fosse soddisfatta questa condizione vedansi i n. 57 e 62 . 
supponiamo inoltre per maggior generalità che

$$
v_{1} \equiv a_{1}, \quad v_{2} \equiv a_{2}, \ldots \quad v_{\eta} \equiv a_{\eta}:
$$

cioè che $n$ punti $v_{k}$ coincidano con altrettanti punti $a_{k}$, il numero $n(\equiv 0)$ potendo essere uguale al minore dei due numeri $q$ e $f_{0}$. [In questo gruppo $(x$ ) i purti $a_{1}, a_{1}, \ldots a_{n}, \quad$ intendono ordinati secondo la grandexa decrescentr delle

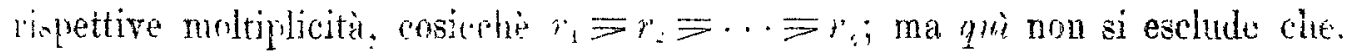
pur essendo $r_{1}$ la molteplicita massima nel gruppo $(n)$, non possa fra gli $f_{0}$ punti fondamentali del sistema esservene taluno, all'infuori del detto gruppo, di moltiplicità maggiore; $\left(C_{\mu}\right)$ essendo d'ordine minimo si avrà però evidentemente a ogni modo $r_{1}+r_{2}+r_{3} ₹ \mu$.]

13. In tale ipotesi, dalla trasformata di una $C_{\mu}$ qualunque si stacca $r_{k}$ volte $(k=1,2, \ldots \eta)$ la $C^{\prime}{ }_{k}$, linea fondamentale del secondo piano corrispondente al punto fondamentale $v_{k}$ e perciò d'ordine $s_{k}$; cosicchè, prescindendo da queste $\eta$ linee fisse $C_{k}^{\prime}$, l'ordine della trasformata $C_{M}$ sarà

$$
M=n \mu-\sum_{k=1}^{k=n} r_{k} s_{k} \text {. }
$$

Inoltre se la $C^{\prime}{ }_{k}$ passa $h_{k i}$ volte pel punto $w_{i}$, la molteplicità $\rho_{i}$ della $C_{M I}$ in $w_{i}$ sarà:

$$
\rho_{i}=\mu \sigma_{i}-\sum_{k=1}^{h} r_{k} r_{k i}
$$

mentre agli $f_{0}-n$ punti $\alpha_{k}$ che sono distinti dai punti $v$ corrispondono nel secondo piano di $T_{n}$ altrettanti punti $a_{k}$, i quali saranno $r_{k}$-pli per la $C_{M H}$.

Onde il sistema dato $\left(C_{\mu}\right)$ si trasforma mediante la $T_{n}$ nel sistema:

$$
\left(C_{H I}\right) \equiv\left[w_{1}^{\rho_{1}} w_{2}^{\rho_{2}} \ldots w_{\varphi}^{\rho_{\varphi}} \mathbf{a}_{n+1}^{r_{n+1}} \ldots \mathbf{a}_{f_{0}}^{r_{f_{0}}}\right],
$$

che appartiene evidentemente alla famiglia considerata.

14. Vogliamo ora determinare il numero $f$ dei punti fondamentali e il numero $f_{c}$ delle linee fondamentali del sistema trasformato $\left(C_{B \prime}\right)$.

Io dico intanto che escluso il caso di $r_{1}+r_{z}=\mu$, nel quale $p_{i}$ può essere nullo, si ha in generale:

$$
\rho_{i}>0, \quad(i=1,2, \ldots \varphi) .
$$

Per dimostrarlo osserro che se $C_{i}$ è la linea fondamentale (d'ordine $\sigma_{i}$ ) del primo piano di $T_{n}$, corrispondente al punto $w_{i}$, il numero $h_{k i}$, oltre che la moltiplicità della $C_{k}^{\prime}$ in $w_{i}$, esprime anche la moltiplicità in $v_{k}$ della $C_{i}-\mathrm{e}$ 
quindi è necessariamente $h_{k i}<\sigma_{i}$, se $\sigma_{i}>1$; e $h_{k i} \equiv 1$ se $\sigma_{i}=1$ : potremo dunque porre:

$$
h_{1 i}=\sigma_{i}-h_{1}, \quad h_{2 i}=\sigma_{i}-h_{2} \quad\left(h_{1} \equiv 0, h_{2} \equiv 0\right) .
$$

Ciò premesso, dal valore (7) di $p_{i}$ è evidente che per $n=0$ e per $n=1$ si ha $\rho_{i}>0$.

Per $\eta=2$, sostituendo le precedenti espressioni di $h_{1 i}$ e $h_{2 i}$ si ha:

onde:

$$
\rho_{i}=\sigma_{i}\left(\mu-r_{1}-r_{2}\right)+h_{1} r_{1}+h_{2} r_{2}
$$

$$
\begin{array}{lll}
\text { se } & r_{1}+r_{2}<\mu, & \rho_{i}>0 ; \\
\text { se' } \quad r_{1}+r_{2}=\mu, & \rho_{i}=0 .
\end{array}
$$

Sia finalmente $n>2$ : dal valore di $\rho_{i}$ si ricava manifestamente:

ossia, ponendo:

$$
p_{i} \equiv \mu \sigma_{i}-r_{1} h_{1 i}-r_{2} h_{2 i}-r_{3}\left[h_{3 i}+\cdots+h_{i n}\right] \text {, }
$$

$$
r_{1}=r_{3}+n_{1}, \quad r_{2}=r_{3}+n_{2} \quad\left(n_{1} \equiv 0, n_{2}=0\right),
$$

e sostituendo poi per $h_{1 i}$ e $h_{2 i}$ le espressioni sopra indicate:

$$
\left.\rho_{i} \equiv \mu \sigma_{i}-r_{3}\left[3 \sigma_{i}-1\right)-\left(h_{\eta+t i}+\cdots+h_{\varphi i}\right)\right]-n_{1}\left(\sigma_{i}-h_{i}\right)-n_{2}\left(\sigma_{i}-h_{2}\right),
$$

0 finalmente:

$$
\rho_{i} \equiv \sigma_{i}\left(\mu-r_{1}-r_{2}-r_{3}\right)+r_{3}\left[1+\sum_{k=a_{i}+1}^{k=p} h_{k i}\right]+\left(r_{1}-r_{3}\right) h_{1}+\left(r_{2}-r_{3}\right) h_{2} .
$$

Ma essendo $r_{3}>0$ (perchè $n>2$ ) ed $r_{1}+r_{2}+r_{3}=\mu$ (perchè il sistema dato $\left(C_{\mu}\right)$ è d'ordine minimo), il secondo membro è un numero positivo; onde a fortiori $\rho_{i}>0$, c. d. d.

Dunque (n..$\left.^{\circ} 13\right)$ se il sistema minimo $\left(C_{\mu}\right)$ è privo di curve fondamentali (teor. I) il numero $f$ dei punti fondamentali del sistema trasformato $\left(C_{M}\right) \grave{e}$ dato da:

$$
f=\varphi+f_{0}-n
$$

15. Supposto $r_{1}+r_{2}=\mu$, nel qual caso il sistema $\left(C_{\mu}\right) \equiv\left[\left[a_{1}^{r_{1}} a_{2}^{r_{2}}\right]\right]_{\mu}$ possiede una retta fondam. $\overline{a_{1} a_{2}}\left(f_{o c}=1\right)$ e due soli punti fondam. $\left(f_{0}=2\right)$, si rileva facilmente da $(\vec{\imath})$ che mediante trasformazioni particolari - per es. mediante la trasformazione quadratica $T_{2} \equiv\left(\begin{array}{lll}a_{1} & a_{2} & v_{3} \\ w_{1} & w_{2} & w_{3}\end{array}\right)$ o mediante la trasformazione. d'ordine $n$ di JONQUIËREs $T_{n} \equiv\left(\begin{array}{lllll}a_{1}^{n-1} & a_{2} & v_{3} & \ldots & v_{\varphi} \\ w_{1}^{n-1} & w_{2} & w_{3} & \ldots & v_{\varphi}\end{array}\right)$ - uno dei $\varphi$ valori $\rho_{i}$ può annullarsi; e quindi in tale ipotesi o si ha l'espressione (9) ove $0 ₹ \eta \rightleftharpoons 2$ oppure si ha $f=\varphi-1+f_{0}-n$ ove $n=2=f_{n}$. 
Cosicchè se il sistema generale minimo $\left(C_{\mu}\right)$ è dotato di una retta fondamentale (teor. I) il numero $f$ dei punti fondamentali del sistema trasformato $\left(C_{\text {Bi }}\right)$ o è espresso da:

o è dato da:

$$
f=\varphi+2-\eta \quad(0 ₹ n ₹ 2),
$$

$$
f=\varphi-1 \quad\left(\eta=f_{0}=2\right) .
$$

16. Indicando genericamente con $C^{\prime}{ }_{j}$ una curva d'ordine $j$, fondamentale pel secondo piano della trasformazione $T_{n}$, e corrispondente a uno dei $\varphi-\eta$ punti $v_{q+1}, \ldots v_{q}$; e con $\alpha_{j i}$ la moltiplicità di $C_{j}^{\prime}$ in $w_{i}$, si ha, come è noto,

$$
j \cdot n=\sum_{i=1}^{i=\sigma_{i}} \sigma_{i} \alpha_{j i}
$$

e mantenendo a $C_{k}^{\prime}$ e ad $h_{k i}$ i significati sopra definiti, si hanno pure le relazioni:

$$
\begin{gathered}
j \cdot s_{1}=\sum_{i=1}^{i=\varphi} \alpha_{j i} h_{1 i} \\
j \cdot s_{2}=\sum_{i=1}^{i=\sum_{\alpha j i}} h_{2 i} \\
\ldots \ldots \\
j \cdot s_{\eta}=\sum_{i=1}^{i=\phi} \alpha_{j i} h_{n i} ;
\end{gathered}
$$

cnde sottraendo da quell' una, moltiplicata per $\mu$, le altre ordinatamente moltiplicate per $r_{1}, r_{2}, \ldots r_{n}$, si ricava:

$$
j\left(n \mu-\sum_{k=1}^{k=0} r_{k} s_{k}\right)=\sum_{i=1}^{i=\alpha_{i j}}\left(\mu \sigma_{i}-\sum_{k} r_{k} h_{k i}\right),
$$

ossia per (6) (7):

$$
j \cdot M=\sum_{i=1}^{i=\beta_{j}} \alpha_{j i} \rho_{i} .
$$

In quest' ultima identità il primo membro essendo uguale al numero totale delle intersezioni di $C_{j}^{\prime}$ con una curva qualunque del sistema trasformato $\left(C_{M}\right)$, e il secondo membro essendo uguale alla somma dei prodotti delle moltiplicità $\alpha_{j i}$ e $\rho_{i}$ di queste due curve in $w_{i}(i=1,2, \ldots \varphi)$, è manifesto che $C_{j}^{\prime}$ e $C_{M}$ non $s^{\prime}$ incontrano all' infuori dei $\varphi$ punti $w_{i}$; onde, rammentando che questi punti sono fondamentali anche per $\left(C_{H H}\right)$, la $C_{j}^{\prime}$ ì una curva fondamentale pel sistema trasformato. E quindi: 
Fra le $\varphi$ linee fondamentali del secondo piano di $T_{n}$ se ne trovano $\varphi-n$, cioè quelle corrispondenti ai punti $v_{\eta+1}, \ldots v_{\varphi}$, che sono fondamentali anche pel sistema $\left(C_{M}\right)$, trasformato di $\left(C_{\mu}\right)$.

17. Tolgasi per un momento la restrizione che $\left(C_{\mu}\right)$ sia un sistema generale e di minimo ordine; e, comunque costituito, lo si supponga dotato di linee fondamentali non spezzantisi in parti. Sia $\Gamma_{\nu}$ (curva di ordine $\nu$ ) una di tali linee; e siano $r_{1}^{\prime}, r_{2}^{\prime}, \ldots r_{\lambda}^{\prime}$ le sue moltiplieità nei punti fondamentali $a_{1}, a_{2}, \ldots a_{\lambda}$ del sistema, dei quali per generalità soltanto $\lambda-\alpha$ si suppongano appartenere al gruppo (n): onde si arrà:

$$
\sum_{h} r_{k} r_{k}^{\prime}=\sum_{k=\alpha} r_{k} r_{k}^{\prime}+\sum_{a} r_{k} r_{k}^{\prime}=\mu \nu
$$

Mediante $T_{n}$, la $\Gamma_{\nu}$ si trasforma in generale in una curva $\Gamma^{\prime}{ }^{\prime}$ dell'ordine:

$$
\nu^{\prime}=n \nu-\sum_{i=\infty} r_{k}^{\prime} s_{k}
$$

avente nel punto $w_{i}$ la moltiplicità:

$$
\rho^{\prime} i=\nu \sigma_{i}-\underset{\lambda=\alpha}{\mathbf{\Sigma}} r_{k}^{\prime} h_{k i}
$$

Il numero totale delle sue intersezioni con una curva qualunque del sistema trasformato $\left(C_{M}\right)$ è dunque $M \nu^{\prime}$, e il numero di quelle assorbite dai punti fondamentali $w_{i}$ e $a_{k}\left(\mathrm{n}^{0}{ }^{0} 13\right)$ è:

$$
\sum_{i=1}^{i=\rho_{i}} \rho_{i} \rho_{i}^{\prime}+\sum_{\alpha} r_{k} r_{k}^{\prime}=\sum_{i=1}^{i=\sum_{p}}\left[\left(\mu \sigma_{i}-\sum_{n} r_{k} h_{k i}\right)\left(\nu \sigma_{i}-\sum_{k=\alpha} r_{k}^{\prime} h_{k i}\right)\right]+\sum_{\alpha} r_{k} r_{k}^{\prime} .
$$

Ma sviluppando, prendendo dalla teorica delle trasformazioni birazionali le ben note relazioni:

$$
\begin{aligned}
& \sum_{i=1}^{i=p_{i}} h_{k i}=n s_{k} \\
& \sum_{i=1}^{i=\varphi} h_{k i}^{2}=s_{k}^{2}+1 \\
& \sum_{i=1}^{i=\varphi} h_{k i} h_{k^{\prime} i}=s_{k} s_{k^{\prime}},
\end{aligned}
$$

e tenendo presente la $(\lambda)$ si ottiene l'identità:

$$
\sum_{i=1}^{i=\rho_{i}} \rho_{i}^{\prime}+\sum_{\alpha} r_{k} r_{k}^{\prime}=\left(n \mu-\sum_{n} r_{k} s_{k}\right)\left(n \nu-\sum_{i=\alpha} r_{k}^{\prime} s_{k}\right)=M \nu^{\prime}
$$


la quale insegna che, quando $\nu^{\prime}$ non si annulla, $\Gamma^{\prime},^{\prime}$ è una linea fondamentale del sistema $\left(C_{M I}\right)$. Dunquo:

II. - Le linee fondamentali di un dato sistema lineare $\left(C_{\mu}\right)$, che non suaniscono per mezzo di successive trasformazioni quadratiohe (o di una determinata trasformazione Cremoniana $T_{n}$ ) dimno luogo a linee fondamentuli del sistena trasformato $\left(C_{3}\right)$; e per conseguenza quando il sistema dato non possiede linee fondamentali, il sistema trasformato non contiene altre curve fondamentali oltre quelle $C_{j}^{\prime}$ indicate al $n .^{\circ} 16$.

18. Supponiamo in particolare $\left(C_{\mu}\right) \equiv\left[\left[a_{1}^{r_{1}} a_{2}^{r_{2}}\right]\right]_{\mu}$ ed $r_{1}+r_{2}=\mu$; in tal caso

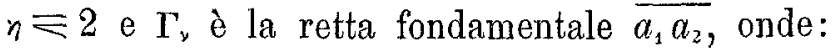

Se

$$
\nu=1, \quad \lambda=2, \quad r_{1}^{\prime}=r_{2}^{\prime}=1 \text {. }
$$

se $\quad n=1, \quad \nu^{\prime}=n-s_{1}>0$;

finalmente se $\quad n=2, \quad \nu^{\prime}=n-s_{1}-s_{2} \equiv 0$.

È chiaro infatti in quest' ultimo caso che, per trasformazioni $T_{n}$ particolari - per es. (n..$^{\circ}$ 15) per la quadratica $T_{2} \equiv\left(\begin{array}{lll}a_{1} & a_{2} & v_{3} \\ w_{1} & w_{2} & v_{3}\end{array}\right)$ o per la trasformazione d'ordine $n$ di JoNQuìres $T_{n} \equiv\left(\begin{array}{lllll}a_{1}^{n-1} & a_{2} & v_{3} & \ldots & v_{\varphi} \\ w_{1}^{n-1} & w_{2} & w_{3} & \ldots & w_{p}\end{array}\right)$ - può $\nu^{\prime}$, ossia $n-s_{1}-s_{2}$, divenire nullo; e quindi, per mezzo della trasformazione, la retta $\overline{a_{1} a_{2}}$ svanisce oppure dà origine a una linea fondamentale di $\left(C_{I I}\right)$.

19. Ritornando al sistema generale minimo $\left(C_{\mu}\right)$ considerato in principio di questo paragrafo, le proprietà testè dimostrate combinate con quella del n. 16 permettono di concludere che: Se il sistema minimo $\left(C_{\mu}\right)$ è privo di curve fondamentali il numero $f_{c}$ delle linee fondamentali del sistema trasformato $\left(C_{\lambda I}\right)$ sarì espresso da:

$$
f_{c}=\varphi-n
$$

e se $r_{1}+r_{2}=\mu$, sarì espresso o $d a$ :

$$
f_{c}=\varphi-n+1 \quad(0 \equiv n \equiv 2),
$$

oppure, quando $n=2$, da:

$$
f_{c}=\varphi-n=\varphi-2 .
$$

20. Dai valori trovati di $f$ e di $f_{c}\left(n{ }^{i} 14,15,19\right)$ si ricava:

1) $\varepsilon \equiv f-f_{c}=f_{0}=f_{0}-0$, se $\left(C_{\mu \nu}\right)$ è privo di linee fondamentali;

2) $\varepsilon \equiv f-f_{c}=1=f_{0}-f_{o c}$, se $\left(C_{\mu}\right) \equiv\left[\left[a_{1}^{r_{1}} a_{2}^{r_{2}}\right]\right]_{\mu=r_{1}+r_{2}}$. 
La differenza $\varepsilon \equiv f-f_{c}$, che chiameremo eccesso degli elementi fondamentali, ha dunque carattere invariantivo per tutt'i sistemi lineari generali soddisfacenti alle condizioni del n. ${ }^{\circ} 11$ e appartenenti a una stessa famiglia. Resta così dimostrato il seguente teorema:

III. - In tutti i sistemi lineari generali e determinati di genere $p$ appartenenti a una stessa famiglia, nei quali due curve qualunque si segano esclusivamente in punti variabili; come pure nei sistemi minori in essi contenuti (n. ${ }^{\circ}$ ), la differenza $f-f_{c} \equiv \varepsilon$ fra il numero dei punti fondamentali arbitrarii e il numero delle linee fondamentali è costante.

Se il sistema generale di minimo ordine $\mu$ corrispondente alla famiglia considerata è privo di linee fondamentali e possiede $f_{0}$ punti fondamentali arbitrarii, quella differenza $\varepsilon$ è uguale ad $f_{0}$; essa è uguale all'unità nel caso contrario (cfr. n. ${ }^{\mathrm{i}} 10$ e 62 ).

21. Di quì si rileva essere l'ordine minimo $\mu$ e l'eccesso $\varepsilon$ due numeri caratteristici per ogni famiglia di sistemi lineari generali. Ad esempio per le seguenti famiglie di reti $\left(c^{\prime}=2\right)$ si ha:

reti omaloidiche $(p=0, l=1) \ldots \quad \varepsilon=0, \quad \mu=1$

reti ellittiche $\left\{\begin{array}{lll}(p=1, k=2) \ldots & \varepsilon=7, & \mu=3 \\ (p=1, k=3) \ldots & \varepsilon=6, & \mu=3 \\ (p=1, k=4) \ldots & \varepsilon=5, & \mu=3 \\ \ldots \ldots \ldots & & \end{array}\right.$

ecc.

22. Come corollario di III si ricava:

IV. - Due sistemi lineari di qualsivoglia genere e dimen. sione, deducibili l'uno dall'altro mediante trasformazioni quadratiche e privi entrambi di curve fondamentali, hanno lo stesso numero $f_{0}$ di punti fondamentali $(*)$.

Si può verificare questo teorema su alcuni degli esempi considerati "nella mia Nota: Di due trasformazioni multiple associate a ogni trasformazione birazionale (Rendic. dei Lincei, 5 dicembre 1886).

(*) Cfr. Caporali, Nota al n. 3 , 1. c. 


\section{§ 4. Classificazione dei sistemi lineari generali d'ordine minimo.}

23. Dal fin qui detto si rileva che per lo studio delle varie famiglie di sistemi lineari di genere $p$ basta limitarsi a considerare i corrispondenti sistemi d'ordine minimo; e di questi ora andiamo ad occuparci.

24. I punti fondamentali semplici influiscono sulla dimensione e sul grado, ma non sull'ordine e sul genere di un sistema generale.

Distingueremo per ciò i sistemi lineari generali di ordine minimo relativi ad un dato genere $p$ in sistemi di tipo normale e sistemi di tipo derivato: chiamando tipo normale un sistema lineare d'ordine minimo $\left(\begin{array}{lll}p & k_{0} & c_{0}^{\prime}\end{array}\right)_{\mu}$ il quale non contenga alcun punto fondamentale semplice, e chiamando tipo derivato ogni altro sistema generale d'ordine minimo $\left(p k l c^{\prime}\right)_{\mu}$.

25. Si passa da un tipo derivato a uno normale (o ad altro derivato) dello stesso genere e di ugual ordine, sopprimendovi tutt'i punti semplici fondamentali (o risp. una parte di essi). E viceversa con l'aggiunta di un conveniente numero di punti fondanentali semplici scelti ad arbitrio, da un dato tipo (normale o derivato) si passa, quando è possibile, ai suoi tipi derivati.

26. Un sistema lineare $\left(C_{\mu}\right)_{p}$ privo di punti fondamentali semplici ̀̀ di ordine minimo ossia è un tipo normale del genere $p$ nei seguenti casi:

(A) Se non ha punti fondamentali multipli;

(B) Se possiede un solo punto multiplo fondamentale;

(C) Se possiede due e due soli punti multipli fondamentali;

(D) Se possiede almeno tre punti multipli fondamentuli, la somma dei cui gradi di moltiplicilà non superi l'ordine $\mu$ del sistema.

$\grave{E}$ evidente infatti che in ciascuna di queste ipotesi la condizione di minimo:

$$
r_{1}+r_{2}+r_{3}=\mu,
$$

è soddisfatta, se, come si suppone, la curva generica $C_{\mu}$. del sistema non si spezza in parti.

27. Avuto speciale rigundo ai tre punti fondamentali di maggior moltiplicitù, classificheremo perciò $\mathrm{i}$ tipi normali (e i derivati) in tipi monomi (ciò contenenti un solo o nessun punto multiplo fondamentale), binomi (cioè conte- 
nenti due e due soli punti multipli fondamentali) e trinomi (ciò contenenti almeno tre punti multipli fondamentali). - In particolare il tipo $\left[\left[a^{\mu-s} b^{s}\right]\right]_{\mu}$ si dirà binomio di $2^{a}$ specie; e i tipi $\left[a_{1}^{m} a_{2}^{m} a_{3}^{m}\right],\left[a^{r} b_{1}^{m} b_{2}^{m}\right],\left[a^{r} b^{s} c^{m}\right]$ si diranno risp. trinomi di $1^{a}, 2^{a}, 3^{a}$ specie $(r \equiv s>m>1)$.

28. Dal n. 25 si ricava poi:

V. - Se $p>0$, ogni tipo normale $\left(p, k_{0}, c_{0}^{\prime}\right)_{\mu}$, tranne quello binomio di $2^{a}$ specie, dà origine in generale a $c_{0}^{\prime}-1$ tipi derivati $\left(p, k, c^{\prime}\right)_{\mu}$; epperò in certo modo rappresenta $c_{0}^{\prime}$ sistemi lineari dello stesso genere $p$ e dello stesso ordine minimo $\mu$, le cui dimensioni e $i$ cui gradi sono rispettivamente:

$$
\begin{array}{llll}
c^{\prime}=1, & 2, \ldots & c_{0}^{\prime}-1, & c_{0}^{\prime} \\
k=p, & p+1, \ldots & k_{0}-1, & k_{0} .
\end{array}
$$

Il tipo normale binomio di $2^{\alpha}$ specie, al contrario, non dà luogo a tipi derivati e rappresenta un unico sistema lineare di ordine minimo $\mu$, di grado $k_{0}$ e di dimensione $c_{0}^{\prime}\left(=k_{0}+1-p\right)$, cioè $i l$ sistema $\left[\left[a^{k-s} b^{s}\right]\right]_{\mu}^{p}$. Aggiungendo anche un solo punto semplice ai suoi punti base, si otterrebbe bensì un altro sistema $\left[a^{\mu-s} b^{s} c \ldots\right]_{\mu}^{p}$ di egual genere $p$ e di ugual ordine $\mu$ (di grado e di dimensione differenti); ma per quest'ultimo evidentemente non sarebbe $\mu$ l'ordine minimo - ossia il nuovo sistema sarebbe riducibile a un tipo (normale o derivato) differente dal dato $\left[\left[a^{\mu-s} b^{s}\right]\right]_{u}^{p}$. - Similmente dal sistema razionale $\left[a^{\mu-1}\right]_{\mu}, \S 5$, Tab. I, b, si ricava un solo tipo derivato, se $\mu>2$.

29. Lo studio di tutti i sistemi generali d'ordine minimo relativi a un dato genere $p$ e a un dato grado $k$, è ricondotto per tal modo alla semplice ricerca dei tipi normali di genere $p$. Per trovare questi tipi normali basterà esaminare ad una ad una le quattro ipotesi del $\mathrm{n} .^{\circ} 26$; il che faremo nei tre paragrafi seguenti.

\section{$\S 5$. Tipi monomi.}

30. Nell'ipotesi $(A)$ n. 26 , equivalente ad $r_{1}=0$, le curve del sistema non potendo avere punti multipli variabili, nè possedendone alcuno di fisso, saranno generali del loro genere (dato) $p$. Ma una curva generale di ordine $\mu$ è del genere $\frac{(\mu-1)(\mu-2)}{2}$; onde posto $\mu-2=h$ dovrà essere:

$$
p=\frac{h(h+1)}{2}, \quad h=-1 \text {. }
$$


Yiceversa se $p$ ha questa forma vi è un sistema lineare, dell'ordine minimo $\mu=h+2$, privo di punti fondamentali: esso è costituito di tutte le curve generali di quest'ordine, situate in un piano; il suo grado è $k_{0}=(h+2)^{2}$, e la sua dimensione $c_{0}^{\prime}=\frac{(h+2)(h+5)}{2}$. Dunque perchè un sistema minimo manchi di punti fondamentali ciò̀ sia del tipo normale monomio [0] necessaria che il suo genere p abbia la forma $(\alpha)$. - Questa è pure la condizione perchè un sistema lineare minimo contenga punti fondamen'ali esclusivamente semplici; infatti un tale sistema è evidentemente un derivato del tipo normale $[0]_{\mu, ~}^{p}$.

31. Nella ipotesi $(B)$ n. ${ }^{\circ} 26$ poniamo $r_{1}=\mu-i$.

Dalle (2) (3) si ricava:

$$
\begin{aligned}
\mu=r_{1}+i & =\frac{2 p+(i-1)(i-2)}{2(i-1)} \\
k=i(2 \mu-i) & =\frac{2 i(p+i-1)}{i-1} \\
r_{1} & =\frac{2 p-(i-1)(i-2)}{2(i-1)} .
\end{aligned}
$$

Dunque:

VI. - Dato p, per ogni soluzione della (a) ossia della:

$$
p=(i-1) r_{1}+\frac{(i-1)(i-2)}{2},
$$

in numeri $i, r_{1}$ interi e positivi si ha un sistema minimo del tipo normale monomio $\left[a^{r_{1}}\right]_{\mu=r_{1}+i}^{p}$.

Se $r_{1}=0$ si ricade sul tipo monomio $\left[a^{0}\right]^{p}, \equiv[0]_{\mu}^{p}$ già considerato nel numero precedente; se $r_{1}=1$ non si ha un sistema minimo di tipo normale, ma uno di tipo derivato dal normale monomio $[0]_{p}^{p}$.

32. Riuniamo nella seguente tabella $\mathrm{i}$ tjpi normali monomi corrispondenti sia ad $r_{1}=0$ sia ai primi cinque valori di $i$. 
di genere qualunque.

Tabelia I. - Tipi nommali monomi $\left[a^{r_{1}}\right]_{\mu}^{p}$.

\begin{tabular}{|c|c|c|c|c|c|c|}
\hline \multicolumn{2}{|c|}{$\begin{array}{c}\text { Simbolo } \\
\text { del sistema }\end{array}$} & $r_{l}$ & $\begin{array}{c}\text { Genere } \\
p\end{array}$ & $\begin{array}{c}\text { Ordine } \\
\text { minimo } \\
\mu\end{array}$ & $\begin{array}{c}\text { Grado } \\
\quad k\end{array}$ & $\begin{array}{c}\text { Dimensione } \\
c^{\prime}=k+1-p\end{array}$ \\
\hline a. & $10]$ & 0 & $p=\frac{\left.h^{\prime} h+1\right)}{2}, h=-1$ & $h+2$ & $(h+2)^{2}$ & $\frac{(h+2)(h+5)}{2}$ \\
\hline b. & {$\left[c^{\mu-1}\right]$} & $\mu-1$ & $p=0$ & $\mu$ & $2 \mu-1$ & $2 \mu$. \\
\hline c. & {$\left[a^{p}\right]$} & $p$ & $p$ & $p+2$ & $4(p+1)$ & $3 p+5$ \\
\hline d. & {$\left[a^{\frac{p-1}{2}}\right]$} & $\frac{p-1}{2}$ & $p=2 h-1, h>1$ & $h+2$ & $3(2 h+1)$ & $4 h+5$ \\
\hline e. & {$\left[a^{\frac{p-3}{3}}\right]$} & $\frac{p-3}{3}$ & $p=3 h, h>1$ & $h+3$ & $8(h+1)$ & $5 h+9$ \\
\hline f. & {$\left[a^{\frac{p-6}{4}}\right]$} & $\frac{p-6}{4}$ & $p=4 h+2, h>1$ & $h+4$ & $5(2 h+3)$ & $6 h+14$ \\
\hline • & & $\cdots$ & $\ldots \ldots$ & $\cdots$ & & \\
\hline
\end{tabular}

33. Per far risaltare il significato di questa tabella, consideriamovi come esempio la prima orizzontale; tenendo presenti i n. ${ }^{\circ} 6$ e 28 se ne ricava:

VII. - Un dato sistema lineare non si può trasformare in uno che sia privo di punti fondamentali oppure possieda punti fondamentali esclusivamente semplici, a meno che $i l$ suo genere non abbia la forma $p=\frac{h(h+1)}{2}$, $h \equiv-1$. Però tutte le volte che $p$ ha questa forma, esistono $c_{0}^{\prime}=\frac{(h+2)(h+5)}{2}$ famiglie di cotali sistemi lineari generali del genere $p$, ma di gradi $k$ differenti; $i$ rispettivi sistemi d'ordine minimo sono rappresentati dal tipo nor-

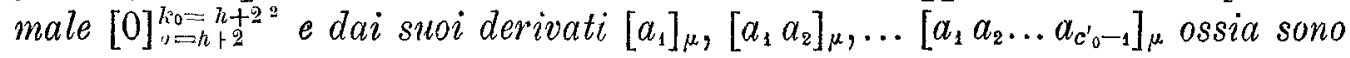
costituiti da sistemi di curve generali dell'ordine $\mu=h+2$, aventi rispettivamente $0,1,2, \ldots \frac{p(\mu+3)}{2}-1$ punti fissi arbitrarii comuni.

Esempi:

per $h=-1$ e per $h=0$ si ha $p=0$ : e si trovano tutti i sistemi minimi costituiti da rette e da coniche; 
generali;

per $h=+1$ si ha $p=1$ : si trovano i nove sistemi minimi di cubiche ecc. ecc.

34. Così dalla terza orizzontale della Tabella I si ricava:

VIII. - Per ogni genere $p$ vi sono $3 p+5$ famiglie di sistemi lineari riducibili rispettivamente ai seguenti sistemi tipici d'ordine minimo $\mu=p+2$, ciò: :

$$
\left[a^{p}\right]_{\mu}^{k_{\varphi}}, \quad\left[\begin{array}{lll}
a^{p} & b_{1}
\end{array}\right]_{\mu}, \quad\left[\begin{array}{llll}
a^{p} & b_{1} & b_{2}
\end{array}\right]_{\mu}, \ldots \quad\left[a^{p} b_{1} b_{2} \ldots b_{3 p+4}\right]_{\mu},
$$

$i$ gradi $k$ e le corrispondenti dimensioni $c^{\prime}$ sono rispettivamente:

$$
\begin{aligned}
& k=4(p+1) \equiv k_{0}, \quad k_{0}-1, \quad k_{0}-2, \ldots \ldots ., \quad p \\
& c^{\prime}=3 p+5 \equiv c_{0}^{\prime}, \quad c_{0}^{\prime}-1, \quad c_{0}^{\prime}-2, \ldots \ldots, \quad 1 \text {. }
\end{aligned}
$$

Per $p=0$ e per $p=1$ si ricade su sistemi riducibili al tipo normale [0] o ai suoi derivati, cioè su sistemi già considerati al numero precedente.

35. Dalla quarta orizzontale, per $p=5$, si ottiene il sistema minimo di tipo normale monomio $\left[a^{2}\right]_{i=3,}^{p=5, k_{0}=24}, c_{0}=17$, il quale ammette altri 16 tipi derivati dello stesso ordine; ecc. ecc.

\section{§. Tipi binomi.}

36. Nell'ipotesi $(C) \mathrm{n}^{\circ} 26$ poniamo:

$$
r_{1}+r_{2}=\mu-i
$$

onde le (2)-(4) somministrano:

$$
\begin{aligned}
& p=r_{1} r_{2}+(i-1)\left(r_{1}+r_{2}\right)+\frac{1}{2}(i-1)(i-2) \\
& k=2 r_{1} r_{2}+i\left[2\left(r_{1}+r_{2}\right)+i\right]=2\left[p+r_{1}+r_{2}+i-1\right]+i \\
& c^{\prime}=k+1-p=p+2\left[r_{1}+r_{2}+i\right]+i-1,
\end{aligned}
$$

e da queste, per $i=0$, si ricava:

IX. - Se $p>0$ ed $r \equiv s>1$, a ogni soluzione in numeri interi dell'equazione $p=(r-1)(s-1)$ corrisponde un tipo normale binomio di $2^{a}$ specie $\left[\left[a^{r} b^{s}\right]\right]_{\text {s. }}^{p}$ dell'ordine $\mu=r+s$, del grado $l_{0}=2 r s$ e della dimensione $c_{0}^{\prime}=$ $=r(s+1)+s$. 


\section{Esempi:}

$p=1$ : tipo normale $\left[\left[a_{1}^{2} a_{2}^{2}\right]\right] ; \mu=4, k_{0}=c_{0}^{\prime}=8$ : curve di $4^{\circ}$ ordine dotate degli stessi due punti doppi e non aventi altre intersezioni fisse comuni.

$p=2: \quad$ tipo normale $\left[\left[a^{3} b^{2}\right]\right] ; \mu=5, k_{0}=12, c_{0}^{\prime}=11:$ curve di $5^{0}$ ordine aventi uno stesso punto triplo, uno stesso punto doppio e nessun altro punto fisso comune.

$p=3: \quad$ tipo normale $\left[\left[a^{4} b^{2}\right]\right] ; \mu=6, k_{0}=16, c_{0}^{\prime}=14:$ curve di $6^{\circ}$ ordine aventi uno stesso punto quadruplo, uno stesso punto doppio e nessun altro punto fisso comune.

$$
\begin{aligned}
& p=4: 1^{\circ} \text { tipo normale }\left[\left[a_{1}^{3} a_{2}^{3}\right]\right] ; \mu=6, k_{0}=18, c_{0}^{\prime}=15 ; \\
& 2^{\circ} \text { tipo normale }\left[\left[a^{5} b^{2}\right]\right] ; \mu=7, k_{0}=20, c_{0}^{\prime}=17 . \\
& p=5: \quad \text { tipo normale }\left[\left[a^{6} b^{2}\right]\right] ; \mu=8, k_{0}=24, c_{0}^{\prime}=20 . \\
& p=6: \quad \text { tipo normale }\left[\left[a^{4} b^{3}\right]\right] ; \mu=7, k_{0}=24, c_{0}^{\prime}=19 . \\
& \text { ecc. ecc. }
\end{aligned}
$$

37. Pel valore $i=1$ si ha dalle (13):

$\mathrm{X}$. - Dato $p$, se $r \equiv s>1$ a ogni soluzione in numeri interi dell'equazione $p=r s$ corrisponde un tipo normale binomio (di $1^{a}$ specie) dell'ordine $\mu=r+s+1$, del grado $k_{0}=2(p+\mu)-1$ e della dimensione $c_{0}^{\prime}=p+2 \mu$.

Esempi:

$p=4: \quad$ tipo normale $\left[a_{1}^{2} a_{2}^{2}\right] ; \mu=5, k_{0}=17, c_{0}^{\prime}=14 ;$

$p=6: \quad$ tipo normale $\left[a^{3} b^{2}\right] ; \mu=6, k_{0}=23, c_{0}^{\prime}=18$;

$p=12: 1^{\circ}$ tipo normale $\left[a^{6} b^{2}\right] ; \mu=9, k_{0}=41, c_{0}^{\prime}=30 ;$

ecc. ecc.

$2^{\circ}$ tipo normale $\left[a^{4} b^{3}\right] ; \mu=8, k_{0}=39, c_{0}^{\prime}=28$;

38. Se nelle (13) si pone $i>1$ si ha:

Onde:

$$
\begin{aligned}
p & >r_{1} r_{2} \\
k & >2\left[p+r_{1}+r_{2}\right]+1 \\
c^{\prime} & >p+2\left[r_{1}+r_{2}+1\right] \\
\mu & >r_{1}+r_{2}+1 .
\end{aligned}
$$

XI. - Dato $p$, se $i>1$ e $r \equiv s>1$, a ogni soluzione in numeri interi della equazione

$$
p=r s+(i-1)(r+s)+\frac{(i-1)(i-2)}{2}
$$

Annali di Matematica, tomo XV. 
corrisponde un tipo binomio di $1^{a}$ specie $\left[a^{r} b^{s}\right]_{\mu}^{p}$ dell'ordine $\mu=r+s+i$, del grado $k=2[p+\mu-1]+i$ e della dimensione $c^{\prime}=p+2 \mu+i-1$. - Questi medesimi tipi normali binomi si trovano pure risolvendo in numeri $r, s, \mu$ interi $(>1)$ le due relazioni:

$$
\begin{aligned}
& p>r s \\
& \mu(\mu-3)=2(p-1)+r(r-1)+s(s-1) .
\end{aligned}
$$

39. Riunendo questi risultati si ha la seguente tabella:

\begin{tabular}{|c|c|c|c|c|c|c|}
\hline \multicolumn{3}{|c|}{$\begin{array}{c}\text { Simbolo e qualifica } \\
\text { del sistema minimo }\end{array}$} & $\begin{array}{c}\text { Genere } \\
\quad p\end{array}$ & $\begin{array}{c}\text { Ordine } \\
\mu\end{array}$ & $\begin{array}{l}\text { Grado } \\
k_{0}\end{array}$ & $\begin{array}{l}\text { Dimensione } \\
c_{0}^{\prime}=k_{0}+1-p\end{array}$ \\
\hline a. & $1^{\mathrm{a}}$ specie & {$\left[a^{r} b^{s}\right]$} & $p>r s$ & $\left.\mu^{\prime} \mu-3\right)=2(p-1)+r(r-1)+s(s-1)$ & $u^{2}-\left(r^{2}+s^{2}\right)$ & $\mu^{2}-\left(r^{2}+s^{2}+p\right)+1$ \\
\hline b. & $1^{a}$ specie & {$\left[a^{\mu-s-1} b^{s}\right]$} & $p=r s$ & $r+s+1$ & $2\left(p+r+s_{j}+1\right.$ & $p+2(r+s+1)$ \\
\hline c. & $2^{\mathrm{a}}$ specie & {$\left[\left[a^{\mu-s} b^{s}\right]\right]$} & $p=(r-1)(s-1)$ & $r+s$ & $2 r s$ & $r(s+1)+s$ \\
\hline
\end{tabular}

Tabella II. - Tipi normali binomi $\left[a^{r} b^{s}\right]_{u}^{p}, \quad(r \equiv s>1)$.

\section{§ 7. Tipi trinomii.}

40. Nell'ipotesi $(D)$ n. 26 poniamo $r_{3}=m>1$.

Siccome $3 m \neq r_{1}+r_{2}+r_{3}$, perchè il sistema sia d'ordine minimo deve essere $\mu \equiv 3 m$. Dunque:

XII. - Indipendentemente dal genere $p$ e dalla dimensione $c^{\prime}$ il numero $3 m\left(=3 r_{3}\right)$ è un limite inferiore per l'ordine $\mu$ dei sistemi lineari minimi del tipo normale trinomio.

41. D'altra parte se si considera un sistema $\left(C_{\mu}\right) \equiv\left(p k c^{\prime}\right)_{\mu}$, generale e determinato dai punti fondamentali $a_{i}$, ma del resto qualunque, dalle (1) (2) (3) si ricava:

$$
\begin{aligned}
& \sum_{4}^{f} r^{2}=\mu^{2}-k-\left(r_{1}^{2}+r_{2}^{2}+r_{3}^{2}\right) \\
& \sum_{4}^{f} r=3 \mu+k-2 c^{\prime}-\left(r_{1}+r_{2}+r_{3}\right)
\end{aligned}
$$


ossia, sottraendo dalla prima la seconda moltiplicata per $r_{3}$,

$$
\begin{gathered}
\sum_{4}^{f} r^{2}-r_{3} \sum_{4}^{f} r= \\
\mu\left(\mu-3 r_{3}\right)-k\left(r_{3}+1\right)-r_{1}^{2}-r_{2}^{2}+r_{3}\left(2 c^{\prime}+r_{1}+r_{2}\right) .
\end{gathered}
$$

Ma il primo membro è nullo se $r_{3}=r_{4}=\cdots=r_{f}$ ed è negativo nel caso contrario; dunque, rammentando che nella ipotesi fatta sussiste la (4), si arrà la:

$$
\mu\left(\mu-3 r_{3}\right)=\left(c^{\prime}+p-1\right)\left(r_{3}+1\right)+r_{1}^{2}+r_{2}^{2}-r_{3}\left[2 c^{\prime}+r_{1}+r_{2}\right],
$$

nella quale, quando $r_{3}<20$ in generale quando $r_{3}=r_{1}=\cdots=r_{f}=m$ qualunque, vale il segno di uguaglianza. Questa relazione sta per qualsivoglia sistema lineare generale e determinato.

42. Supposto ora che:

$$
r_{1}+r_{2}+r_{s_{2}} \approx \mu
$$

dalla precedente si avrà a fortiori la:

$$
2\left(r_{1} r_{2}-r_{3}^{2}\right)=\left(p-1+c^{\prime}\right)\left(r_{3}+1\right)-2 c^{\prime} r_{3}
$$

che si può anche scrivere nella forma:

$$
2\left(r_{1} r_{2}-r_{3}^{2}\right)+\left(r_{3}-1\right)\left(c^{\prime}-p+1\right)=2(p-1),
$$

ed è la relazione di condizione perchè un sistema lineare (generale e determinato) sia d'ordine minimo.

43. Ritornando all'ipotesi $(D)$ poniamo ancora $r_{3}=m>1$. La quantità $c^{\prime}-p+1$ ha il valor massimo, compatibilmente con la condizione (15), quando $r_{3}$ e $2\left(r_{1} r_{2}-r_{3}^{2}\right)$ hanno i loro valori minimi 2 e 0 , cioè quando $r_{1}=r_{2}=r_{3}=2$; in quest' jpotesi l'anzidetta condizione diviene:

dalla quale per (4):

$$
c^{\prime}=3(p-1),
$$

$$
k=₹ 4(p-1) \text {. }
$$

Dunque $i$ numeri $3(p-1)$ e $4(p-1)$ sono risp. limiti superiori per la dimensione $e^{\prime}$ e pel grado $k$ dei sistemi lineari generali di minimo ordine $e$ del tipo normale trinomio.

44. Risulta inoltre da quanto precede che $\mathrm{i}$ sistemi generali minimi dí tipo normale trinomio relativi a un dato genere $p$ si ottengono risolvendo in 
numeri interi $\mu, c^{\prime}, r_{1}, r_{2}, r_{3}$ le due relazioni $(14),(15)\left(^{*}\right) . \mathrm{Ma}$ anche tenuto conto (come è necessario) delle condizioni restrittive:

$$
c^{\prime}=3(p-1), \quad \mu \equiv 3 r_{3}, \quad r_{1} \equiv r_{2} \equiv r_{3}>1,
$$

non ogni soluzione intiera delle relazioni anzidette dà luogo a un sistema minimo di tipo trinomio. Trovati infatti, mediante le medesime, l'ordine, le tre moltiplicità più elevate dei punti fondamentali e fra certi limiti la dimensione del sistema, bisogna per mezzo delle (1)-(3) completare (quando è possibile) la determinazione delle moltiplicità degli altri suoi punti fondamentali e di tutte le sue caratteristiche. Sotto la doppia riserva che ciò sia aritmeticamente possibile e che inoltre $\mathrm{i}$ valori così trovati non siano geometricamente incompatibili (cioè definiscano delle vere curve algebriche formanti un sistema lineare) diremo soluzione geometrica la soluzione considerata delle (14) (15).

Dunque concludendo:

XIII. - Dato p, a ogni soluzione geometrica delle (14) (15) corrisponde un sistema lineare generale d'ordine minimo del tipo normale trinomio.

45. A meglio chiarire il numero precedente consideriamo come esempio i sistemi normali trinomii di $1^{a}$ specie.

Le (14) (15) ammettono la soluzione:

essendo:

$$
r_{1}=r_{2}=r_{3}=m, \quad \mu=3 m,
$$

e:

$$
m \text { qualunque, se } c^{\prime} ₹ p-1 \text {, }
$$

$$
m=2 p-1, \text { se } c^{\prime}>p-1 ;
$$

perchè questa sia, nel senso testè spiegato, una soluzione geometrica delle relazioni anzidette bisogna inoltre:

1. ${ }^{\circ}$ che siano soddisfatte anche le due condizioni:

$$
\left.\begin{array}{l}
\frac{3 m(3 m-3)}{2}-(p-1)=\frac{m(m-1)}{2} \alpha_{m}+\cdots+3 \alpha_{3}+\alpha_{2} \\
\frac{3 m(3 m+3)}{2}-c^{\prime} \quad=\frac{m(m+1)}{2} \alpha_{m}+\cdots+6 \alpha_{3}+3 \alpha_{2},
\end{array}\right\}
$$

dalle quali si ricavano il numero totale $\alpha_{m}(\equiv 3)$ dei punti fondamentali $m$-pli e quelli $\alpha_{2}, \alpha_{3}, \ldots \alpha_{r}$ dei punti fondamentali doppi, tripli,...r-pli;

(*) È chiaro che queste stesse relazioni possono servire anche alla determinazione dei tipi normali monomii e binomii. Si arrebbe, applicandole, la conferma dei risultati da noi già conseguiti per altra via nei due paragrafi precedentí. 
2. ${ }^{\circ}$ che sia riconosciuta la compatibilità geometrica dei valori trovati.

Verificate queste condizioni e supposto $m>1$ si ha un sistema generale minimo del tipo normale trinomio di $1^{a}$ specie, cioè :

$$
\left[a_{1}^{m} a_{2}^{m} a_{3}^{m} \ldots a_{\alpha_{m}}^{m} b_{1}^{m-1} \ldots b_{\alpha_{m},}^{m-1} \ldots d_{1}^{3} \ldots d_{\alpha_{3}}^{3} e_{1}^{2} \ldots e_{\alpha_{2}}^{2}\right]_{\mu=3 m}^{p} \text {. }
$$

46. Altri tipi trinomii di $1 .^{a}$ specie si ricavano dalla seguente soluzione:

$$
r_{1}=r_{2}=r_{3}=m>1, \quad \mu=3 m+h \quad(h>0)
$$

delle (14) (15), che è vincolata alla condizione:

$$
h(3 m+h)+c^{\prime}(m-1)=(p-1)(m+1) .
$$

Questa non ammette soluzioni se $p<4$. Se $p=4$, non ne ammette per $m>3$; e per $m=3$ dà le due soluzioni $m=2, \mu=7$ ed $m=3, \mu=10$ : - delle quali però soltanto la prima è una soluzione geometrica e somministra un tipo normale trinomio di $1^{\mathrm{a}}$ specie, cioè la rete:

$$
\left(C_{7}\right) \equiv\left[a_{1}^{2} a_{2}^{2} \ldots a_{11}^{2}\right]_{\mu=7, h=5}^{p=h, c=5}, c^{\prime}=2,
$$

47. Posto $r_{1}=r_{3}+\eta_{1}, r_{2}=r_{3}+\eta_{2}$ le (14) (15) divengono:

$$
\begin{aligned}
& \mu\left(\mu-3 r_{3}\right)=(p-1)\left(r_{3}+1\right)-c^{\prime}\left(r_{3}-1\right)+r_{1} n_{1}+r_{2} \eta_{2}, \\
& 2 r_{3}\left(\eta_{1}+\eta_{2}\right)+2 \eta_{1} \eta_{2}+\left(r_{3}-1\right)\left(c^{\prime}-p+1\right) \rightleftharpoons 2(p-1),
\end{aligned}
$$

nella qual forma meglio si prestano per la ricerca dei tipi normali trinomii di $2^{a}$ e di $3^{a}$ specie:

pei tipi di $2^{\text {a }}$ specie essendo per lo meno $r_{1}+r_{2}+r_{3}=3 m+1$ $\left(r_{3}=m>1\right)$ si ha $\mu=3 m+1$;

per quelli di $3^{a}$ specie essendo per lo meno $r_{1}+r_{2}+r_{3}=3 m+2$ $\left(r_{3}=m>1\right)$ si ha $\mu>3 m+2$.

Se $c^{\prime} \equiv p-1$ viene:

$$
r_{3}\left(n_{1}+n_{2}\right)+n_{1} n_{2}=p-1
$$

e quindi pei trinomii di $2^{a}$ specie $\left(n_{1}>0, n_{2}=0\right)$ :

$$
1<r_{3} \equiv p-1 \text {, }
$$

e pei trinomii di $3^{a}$ specie $\left(n_{1}>0, n_{2}>0\right)$ :

$$
1<r_{3}=\frac{p-2}{2} \text {. }
$$

Se $c^{\prime}<p-1$, posto $c^{\prime}=p-1-h(1 ₹ h \equiv p-2)$ viene:

$$
2 r_{3}\left(n_{1}+n_{2}\right)+2{y_{1} n_{2}} \equiv 2(p-1)+h\left(r_{3}-1\right) \text {. }
$$

48. I pochi risultati generali relativi ai tipi normali trinomii possono riassumersi nella seguente tabella: 
Tabeita III. - Tipi normali trinomi $\left[a^{r} b^{s} c^{m} \ldots\right], r \equiv s \equiv m>1$.

\begin{tabular}{|c|c|c|c|c|c|c|}
\hline \multicolumn{3}{|c|}{$\begin{array}{c}\text { Simbolo e qualifica } \\
\text { del sistema minimo }\end{array}$} & $\begin{array}{c}\text { Ordine } \\
\mu\end{array}$ & $\begin{array}{c}\text { Genere } \\
p\end{array}$ & $\begin{array}{c}\text { Grado } \\
k_{0}\end{array}$ & $\begin{array}{c}\text { Dimensione } \\
c_{0}^{\prime}\end{array}$ \\
\hline a. & $1^{a}$ specie & {$\left[a_{1}^{m} a_{2}^{m} a_{3}^{m} \ldots\right] m>1$} & $\mu>3 m$ & & & \\
\hline b. & $2^{x^{\prime}}$ specie & {$\left[a^{r} b_{1}^{m} b_{2}^{m} \ldots\right] \quad r>m>1$} & $\mu>3 m+1$ & $p>1$ & $k_{0}=4(p-1)$ & $c_{0}^{\prime}<3(p-1)$ \\
\hline c. & $3^{a}$ specie & {$\left[a^{r} b^{s} c^{m} \ldots\right] r>s>m>1$} & $\mu>3 m+2$ & 11 & & \\
\hline d. $(*)$ & $1^{\mathrm{a}}$ specie & {$\left[a_{1}^{p} a_{2}^{p} \ldots a_{8}^{p} \iota^{p-1}\right]$} & $\mu=3 p$ & $p>0$ & $2 p-1$ & $p$ \\
\hline
\end{tabular}

\section{$\S 8$. Alcuni teoremi generali,}

49. Tenuto presente il $\mathrm{n}^{\circ} 6$ si ha:

$\mathrm{XIV}$. - Un sistema di curve dell'ordine $M \equiv 3 m$ e di genere $p>0$, dotato di almeno tre punti fondamentali (arbitrarii o non arbitrarii) $m$-pli $(m \equiv 1)$ è un sistema lineare del minimo ordine.

Ad esempio sono d'ordine minimo:

(a) i sistemi di cubiche (generali) passanti per tre o più di tre punti fissi arbitrarii $(p=1)\left({ }^{*}\right)$;

(b) i sistemi di $C_{6}$ aventi 8 punti doppi comuni e passanti per $0,1,2$ punti arbitrarii $(p=2)$;

(c) i sistemi di $C_{6}$ aventi 7 punti doppi comuni e passanti per $0,1, \ldots 5$ punti fissi $(p=3)$;

(d) i sistemi di $C_{9}$ aventi 8 punti tripli e 1 punto doppio comuni e passanti per $0,1,2$ punti fissi $(p==3)$,

(e) i sistemi di $C_{6}$ aventi 6 punti doppi comuni e passanti per $0,1,2, \ldots 8$ punti fissi $(p=4)$;

$(f)$ i sistemi di $C_{12}$ aventi 8 punti quadrupli e 1 punto triplo comuni, e passanti per $0,1,2,3$ punti fissi $(p=4)$;

ecc. ecc.

(*) Vedi la nota (*) al n. $^{\circ} 67$.

(**) Si è veduto del resto per altra via $\left(n *^{\circ} 33\right)$ che qualunque sistema di cubiche generali è d'ordine minimo. 
50. Le relazioni (16) del $n^{\circ} 45$ si possono scrivere nella forma:

$$
\left.\begin{array}{l}
{\left[9-\alpha_{m}\right] m(m-1)-(m-1)(m-2) \alpha_{m-1}-\cdots-6 \alpha_{3}-2 \alpha_{2}=2(p-1)} \\
{\left[9-\alpha_{m}\right] m(m+1)-\quad m(m-1) \alpha_{m-1}-\cdots-12 \alpha_{3}-6 \alpha_{2}=2 c^{\prime}}
\end{array}\right\}
$$

dalla quale si rileva che se $p>1, \alpha_{m}<9$; onde:

$\mathrm{XV}$. - Il massimo numero di punti fondamentali m-pli per un sistema minimo generale di curve d'ordine $\mu=3 m$ e di genere $p>1 \grave{e} \alpha_{m}=8$; se $p=1$, il valore $\alpha_{m}=9$ soddisfa la prima (16) ma non la seconda (perchè $c^{\prime}$ dev'essere per lo meno $=1$ ); onde:

XVI. - Non esiste alcun sistema generale di curve d'ordine $\mu=3 m$ dotate di 9 punti m-pli comuni; se un sistema lineare di tali curve esiste, esso è necessariamente del genere uno e $i$ suoi punti fondamentali non hanno posizioni del tutto arbitrarie.

$\mathrm{E}$ infatti per ogni valore intero di $m$ vi è un fascio di curve ellittiche di ordine $\mu=3 m$, dotate di 9 punti $m$-pli comuni $\left(c^{\prime}=1, p=1, k=0\right)$; curve studiate per la prima volta dal sig. HaLPHen (nel Bulletin de la Société Mathématique de France, t. $\mathrm{X}_{6}$, pag. 162-172). Se $m>1$ i 9 punti $m$-pli sono situati in una e una sola cubica [che è evidentemente una linea fondamentale $\left.\left(n .^{\circ} 5\right)\right]$ ed 8 di essi non determinano univocamente il rimanente; onde il fascio è del minimo ordine $\left(n .^{\circ} 49\right)$ ed è un sistema speciale di $2^{\circ}$ tipo $\left(\mathrm{n}^{\circ} 55\right)$; mentre se $m=1$ esso è un sistema minimo speciale di $1^{\circ}$ tipo (n. $.^{\text {i }} 54-56$ ).

51. Riguardo ai sistemi riducibili al tipo normale trinomio, dal n..$^{\circ} 43$ si ha:

XVII. - Nessun sistema lineare generale di genere $p$ il cui grado $k$ sia maggiore di $4(p-1)$ è trasformabile in altro d'ordine minimo del tipo normale trinomio;

o ch'è lo stesso:

XVIII. - Soltanto $i$ sistemi lineari di genere $p, e$ di dimensione $c^{\prime}$ non superiore a $3(p-1)$, possono ridursi a sistemi generali d'ordine minimo di tipo trinomio.

Quando $p=0$ o $p=1$ questo limite $3(p-1)$ è sempre superato da $c^{\prime}$; quindi:

XIX. - Per $p=0$ e $p=1$ non vi sono sistemi generali d'ordine minimo del tipo normale trinomio.

Onde il teorema:

XX. - Qualunque sistema lineare di genere zero e qualunque sistema lineare generale di genere uno è trasformabile in altro d'ordine minimo del tipo monomio o binomio (v. $\S 10$, Tabella IV). 
E come primo corollario:

XXI. - In ogni sistema lineare di genere zero, avente più di due punti fondamentali, la somma dei tre gradi più elevati di moltiplicità supera l'ordine $M$ del sistema; cosicchè $r_{1}+r_{2}+r_{3}>M$.

Di questo teorema (già noto) si ha così una nuova dimostrazione; la quale, perchè fondata sulla ricerca diretta di tutti i sistemi generali di minimo ordine corrispondenti a un dato genere $p$, pare più spontanea della usuale $\left(^{*}\right)$.

Osservando poi che dal tipo normale monomio $[0]_{p=3}^{p=1}(\S 10$, Tabella IV) si ricavano $\mathrm{i}$ tipi derivati:

$$
\left[a_{1} a_{2} a_{3}\right]_{\mu=3}, \quad\left[a_{1} a_{2} a_{3} a_{4}\right]_{\mu=4}, \ldots \ldots
$$

si ha come secondo corollario questo nuovo teorema:

XXII. - In ogni sistema lineare generale di genere uno e di ordine $M>3$, se vi sono più di due punti fondamentali, la somma dei tre gradi più elevati di moltiplicità supera $M$, cosicchè $r_{1}+r_{2}+r_{3}>M$ (v. n. 50 e 65 ).

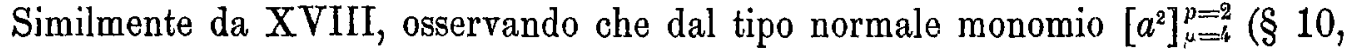
Tabella IV) si ricavano i derivati :

si ha:

$$
\left[a^{2} b_{1} b_{2}\right]_{\mu=4}, \quad\left[a^{2} b_{1} b_{2} b_{3}\right]_{\mu=4}, \ldots
$$

XXIII. - In ogni sistema lineare generale di genere $p=2$, di dimensione $c^{\prime}>3$ e di ordine $M>4$, il quale possieda più di due punti fondamentali, sarà $r_{1}+r_{2}+r_{3}>M$.

52. Terminiamo questo paragrafo con una notevole proprietà dei sistemi lineari riducibili al tipo normale [0], la quale è un semplice corollario del teorema III (§ 3).

XXIV. - Nei sistemi lineari generali riducibili al tipo normale $[0]_{\mu}(* *)$, e generalmente in questi soltanto, il numero dei punti base è uguale al numero delle linee fondamentali.

(*) NoEther, Ueber Flächen, welche Schaaren rationaler Curven besitzen, Math. Ann,, t. III, pag. 165 - CIEBsCH-LindemanN, Vorlesungen über Geometrie, Lipsia, 1875. t. I, pag. 488. - G. B. Guccia, Generalizzazione di un teorema di Noether, Rendic. Circolo matem. di Palermo. t. $\mathrm{I}_{3}$ (1886), pag. 141.

(**) Non ai derivati del tipo normale $[0]_{\mu}$. 
Se fra queste si trovano $\alpha_{1}$ rette, $\alpha_{2}$ coniche, $\alpha_{3}$ cubiche,... si troveranno fra quelli $\beta_{1}$ punti $\mu$-pli, $\beta_{2}$ punti $2 \mu$-pli, $\beta_{3}$ punti $3 \mu$-pli, $\ldots$; e i numeri $\beta$ saranno uguali ai numeri $\alpha$ presi generalmente in ordine diverso.

Per $\mu=1$ si ricade su una nota proprietà delle reti omaloidiche.

\section{§9. Sistemi lineari speciali.}

53. Se i punti fondamentali non sono dati ad arbitrio come finora si era supposto, ma hanno posizioni speciali o fra loro dipendenti, il sistema lineare $\left(C_{n}\right)$, definito al $\S 1$ non è generale, ma è un sistema lineare speciale.

54. Può in tal caso avvenire che alcuni punti fondamentali (scelti ad arbitrio) determinino univocamente le posizioni degli altri (punti fondamentali dipendenti): assunti come punti fondamentali, si trovano ad esempio in queste condizioni i 9 punti base di un fascio di cubiche; i 10 punti base di una rete di sestiche aventi due punti semplici e otto doppi comuni $\left({ }^{*}\right)$; ecc.

55. Se invece avviene il contrario, i punti fondamentali non si possono generalmente distinguere gli uni dagli altri: per esempio i 9 punti $m$-pli di un fascio di $C_{3 m}$, presi come punti fondamentali, si trovano in queste condizioni.

56. Corrispondentemente alle due ipotesi accennate, $\mathrm{i}$ sistemi lineari speciali si diranno del $1^{\circ}$ e del $2^{\circ}$ tipo.

57. I sistemi speciali di $1^{\circ}$ tipo possono ricavarsi dai sistemi generali studiati nei paragrafi precedenti, nei quali in sostanza essi sono impliciti.

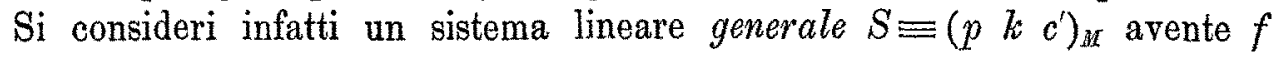
punti fondamentali arbitrarii $a_{1}, a_{2}, \ldots a_{f}$ e si supponga (n. $\left.{ }^{0} 1\right)$ che nel gruppo (k) delle intersezioni di due $C_{M}$ qualunque, esterne ai punti $a_{i}$, si trovino $k^{\prime \prime}(>0)$ punti fissi e $k^{\prime}$ punti variabili - cosicchè sia $\beta=f+k^{\prime \prime}$ il numero totale dei suoi punti base. Se questi $\beta$ punti base $a_{1}, a_{2}, \ldots a_{f}, a_{f+3}, \ldots a_{\beta}$ si

(*) 亡̀ noto (Bertinr, Ricerche sulle trasformazioni univoche iuvolutorie, $\mathrm{n}^{\circ} 35, \mathrm{An}$. nali di Matematica, Ser. $2^{\mathrm{a}}$, t. VIII) ehe le curve di $6^{\circ}$ ordine aventi 8 punti doppi comuni e passanti per un nono punto dato ad arbitrio, passano pure per un decimo punto determinato; e che i 10 punti sono situati in una medesima cubica. Tali curve del resto formano un sistema lineare che rientra nel sistema generale $\left(\mathrm{n} .{ }^{\circ} 48\right.$, Tabella III, d.) di tipo trinomio $\left\langle C_{3 p}, p \equiv\left[a_{1}^{p} a_{2}^{p} \ldots a_{3}^{p} 乙^{p-1}\right]_{c^{\prime}=p}^{k=2 p-1} ;\right.$ e quindi gode della proprietà di questo sistema [r. la nota $\left.{ }^{*}\right)$ al n..$\left.^{\circ} 67\right]$.

Annali di Matematica, tomo XV. 
riguardano ora come punti fondamentali dati, poichè non sono tutti arbitrarii, essi determinano evidentemente un sistema lineare speciale $S^{\prime \prime} \equiv\left(p, k^{\prime}, c^{\prime}\right)_{H}$ dello stesso genere, ordine e dimensione di $S$; il quale però può contenere linee fondamentali, anche se $S$ non ne possiede, e nel quale il grado $k\left(\equiv k^{\prime}\right.$ ) esprime esclusivamente il numero delle intersezioni variabili di due $C_{M}$ qualunque ed ha carattere invariantivo.

Così ad esempio un fascio di curve generali del genere $p$ è un sistema lineare speciale di $1^{0}$ tipo o è un sistema generale di grado $k=p$, secondo che vi si considerano come fondamentali tutt'i punti base o soltanto quelli che si possono prendere ad arbitrio; un fascio di sestiche aventi 8 punti doppi e 4 punti semplici comuni è un sistema speciale di $1^{\circ}$ tipo dotato di due cubiche fondamentali o ̀̀ un sistema generale di grado $k=p=2$, secondo che vi si considerano come fondamentali tutt'i punti base o soltanto i 10 che si possono prendere ad arbitrio.

58. Fra le caratteristiche del sistema speciale $S^{\prime}$ ha luogo la relazione:

$$
c^{\prime}>k+1-p
$$

Infatti le caratteristiche del sistema generale $S$ sono legate dalla relazione (4) $\S 1$ ossia $c^{\prime}=k^{\prime}+k^{\prime \prime}+1-p$, che per $k^{\prime} \equiv k$ e $k^{\prime \prime}>0$ conduce alla (4)".

59. Un sistema lineare il cui grado $k$ esprima esclusivamente punti variabili è necessariamente speciale se $c^{\prime}>k+1-p$; e pù̀ essere speciale se $c^{\prime}=k+1-p$.

Infatti, per l'jpotesi qui fatta su $k$, tutt'i punti base sono punti fondamentali.

Ora nel primo caso $\left(c^{\prime}>k+1-p\right)$ i punti fondamentali non possono essere tutti arbitrarii, perchè se fossero si avrebbe $\left(\mathrm{n}^{\circ} 2\right)$, contro l'ipotesi, $c^{\prime}=k+1-p$.

Nel secondo caso $\left(c^{\prime}=k+1-p\right)$ potendosi disporre ad arbitrio di tutti i punti fondamentali, niente impedisce di assumerli in posizioni speciali. Cosi, ad esempio, se $c^{\prime}=2, k=3, p=2$, uno dei sistemi d'ordine minimo è rappresentato dalla rete:

$$
\left(C_{4}\right) \equiv\left[a^{2} b_{1} b_{2} \ldots b_{9}\right]
$$

la quale è un sistema minimo generale, e per ćiò privo di linee fondamentali, se i 10 punti fondamentali si prendono (com'è lecito) in posizioni arbitrarie; ed è un sistema speciale minimo, dotato di tre coniche fondamentali:

$$
\begin{aligned}
& \Gamma_{2} \equiv a b_{1} b_{2} b_{3} b_{4} b_{5} b_{6} \\
& \Gamma^{\prime}{ }_{2} \equiv a b_{1} b_{2} b_{3} b_{7} b_{8} b_{9} \\
& \Gamma_{2}^{\prime \prime} \equiv a b_{4} b_{5} b_{6} b_{7} b_{8} b_{9},
\end{aligned}
$$


se i punti fondamentali $a, b_{1}, b_{2}, \ldots b_{6}$ si prendono (com'è lecito) sopra una conica $\Gamma_{2}$, e per $b_{7}, b_{8}, b_{9}$, si assumono le ulteriori intersezioni di due coniche $\Gamma_{2}^{\prime}, \Gamma_{2}^{\prime \prime}$, passanti l'una per $a, b_{1}, b_{2}, b_{3}$, l'altra per $a, b_{4}, b_{5}, b_{6} .-\mathrm{E}$ altri sistemi speciali si ricaverebbero specializzando altrimenti le posizioni dei 10 punti fondamentali.

60. Un sistema lineare minimo dotato di una o più linee fondamentali, $e$ che non sia del tipo normale binomio, $\dot{e}$ un sistema lineare speciale. Infatti se i punti fondamentali fossero arbitrarii $\left(\mathbf{n}^{\circ}{ }^{10}\right.$ ) il sistema non conterrebbe (contro l'ipotesi) alcuna linea fondamentale.

61. Sia $\left(C_{\mu}\right)$ un sistema lineare speciale del minimo ordine e $\Gamma_{v}$ una sua curva fondamentale. Se si applica la trasformazione $T_{n}$ indicata al $n .^{\circ} 12$ e si mantengono le stesse notazioni adoperate ivi e al n. ${ }^{\circ} 17$; riflettendo che il sistema qui considerato non è del tipo binomio di $2^{a}$ specie, si arrà $p_{i}>0$, come si è dimostrato al n. ${ }^{\circ} 14$. E supposto che $\Gamma_{v}$ sia una curva fondamentale minima $\left(^{*}\right)$ si dimostra nello stesso modo essere $\rho^{\prime} i>0$; cosicchè l'espressione $\sum_{\alpha} r_{k} r_{k}^{\prime}+\sum_{i=1}^{i=\phi} \rho_{i} \rho_{i}^{\prime}$ è una quantità positiva e quindi da (12) si ricava $\nu^{\prime}>0$. In altri termini:

XXV. - Le linee fondamentali minime di un sistema lineare speciale del minimo ordine non sraniscono per mezzo di successive trasformazioni quadratiche o di una trasformazione cremoniana qualsivoglia.

Ad esempio comunque si trasformi la rete d'ordine minimo:

$$
\left(C_{6}\right) \equiv\left[\begin{array}{lllll}
a_{1}^{2} & a_{2}^{2} \ldots & a_{8}^{2} & b_{1} & b^{\prime}{ }_{1}
\end{array}\right]_{\mu=6, c^{\prime}=2}^{p=2},
$$

dotata della cubica fondamentale minima $\Gamma_{3} \equiv a_{1} \ldots a_{8} b_{1} b_{1}^{\prime}$ si otterrà una rete contenente almeno una curra fondamentale; comunque si trasformi il fascio d'ordine minimo:

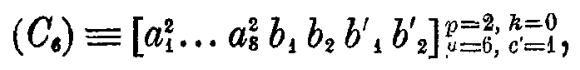

dotato delle due cubiche fondamentali minime:

$$
\Gamma_{3} \equiv a_{1} \ldots a_{8} b_{1} b_{1}^{\prime}, \quad \Gamma_{3}^{\prime} \equiv a_{1} \ldots a_{8} b_{2} b_{2}^{\prime},
$$

(*) Per brevità chiamo minima una curva fondamentale d'ordine $\nu>1$, quando la somma delle tre sue moltiplicità più elevate (nei punti fondamentali del sistema) non ne superi l'ordine $v$. 
si otterrà un fascio dotato di almeno due curve fondamentali; comunque si trasformi il fascio d'ordine minimo:

$$
\left(C_{3 m}\right) \equiv\left[a_{1}^{m} a_{2}^{m} \ldots a_{9}^{m}\right]_{\mu=3 m,}^{p=1,} \begin{gathered}
k=0 \\
e^{\prime}=1
\end{gathered},
$$

dotato (se $m>1$ ) della cubica fondamentale minima $\Gamma_{3} \equiv a_{1} a_{2} \ldots a_{9}\left(\mathrm{n}^{0} 50\right)$, si otterrà un fascio dotato di almeno una curva fondamentale; ecc.

62. Segue dal teorema testè dimostrato che se $\left(C_{\mu}\right)$ possiede $\beta$ punti base e $\gamma(\equiv 0)$ curve fondamentali minime, il sistema trasformato $\left(C_{\mu}\right)$ conterrà $\left(\mathrm{n} .{ }^{\circ} 14\right)$ :

punti base e (n. $\left.{ }^{\circ} 19\right)$ :

$$
f=\varphi-n+\beta
$$

$$
f_{c}=\varphi-n+\gamma,
$$

linee fondamentali; e per conseguenza l'eccesso degli elementi fondamentali di $\left(C_{M}\right)$ sarà :

$$
\varepsilon \equiv f-f_{c}=\beta-\gamma .
$$

Onde tenuto presente il teorema III e il corollario di II ( $(3)$ si può ora dire più generalmente:

XXVI. - Se un sistema lineare di genere $p$ è riducibile a un sistema minimo privo di curve fondamentali (*) o a uno dotato di una sola retta fondamentale o a uno dotato di $\gamma$ linee fondamentali minime, l'eccesso $\varepsilon$ sarà rispettivamente $=\beta 0=10=\beta-\gamma ; \beta$ indicando il numero dei punti base del sistema minimo.

\section{$\S 10$. Sistemi generali d'ordine minimo dei generi $p=0,1,2$.}

63. In questo paragrafo diamo pei primi tre generi i sistemi generali minimi del tipo normale e $\mathrm{i}$ corrispondenti tipi derivati, come li abbiamo ottenuti applicando $\mathrm{i}$ metodi precedentemente esposti.

Notiamo però che, per quanto è detto ai n.i 54,57 e 66 xxxIr, i derivati del terzo sistema normale di genere $p=2$ contengono implicitamente due sistemi minimi speciali di $1^{o}$ tipo, ciò̀ la reté e il fascio di sestiche indicati negli esempii del $n .^{\circ} 61$.

(*) Fanno eccezione i fasci del genere zero. Per un fascio di curve razionali ritengo aver luogo invece la relazione $\gamma=2(\beta-1)$ : la quale trovai verificata in tutti gli esempi che mi è accaduto di considerare. 
di genere qualunque.

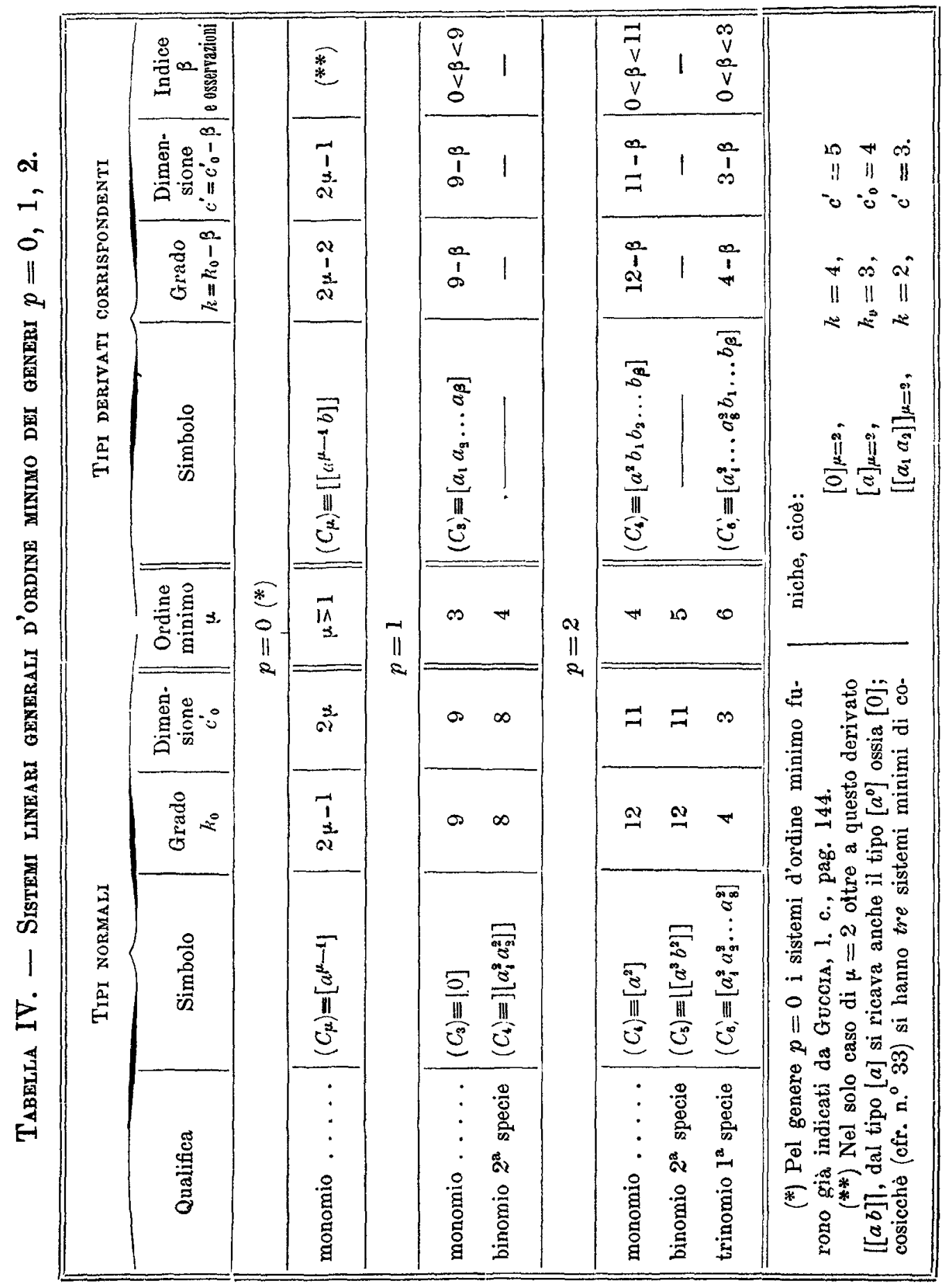


Aggiungeremo per mettere meglio in rilievo alcune delle proprietà condensate in questa tabelia, le seguenti osservazioni relative a ciascun genere.

64. Genere $p=0$.

Per $k=4$ vi sono due famiglie di sistemi lineari del genere zero:

(a) quelli della prima famiglia sono riducibili alla serie $\infty^{5}$ di coniche $[0]_{\mu=2}$ poste in un piano $e$ il rispettivo eccesso $\varepsilon \grave{e}=0$;

(b) quelli dell'altra sono riducibili alla serie $\infty^{5}$ di cubiche $\left[\left[a^{2} b\right]\right]_{\mu=3}$ aventi un punto doppio e un punto semplice comuni; il rispettivo eccesso $\varepsilon$ $\grave{e}=1$.

Per qualsivoglia altro valore del grado $k$ vi è una sola famiglia di sistemi lineari razionali.

XXVII. - In ogni rete omaloidica $\left(^{*}\right)\left(k=1, c^{\prime}=2\right)-e$ in ogni sistema lineare appartenente all'anzidetta prima famiglia $\left(k=4, c^{\prime}=5\right)-$ vi sono tante curve fondamentali quanti sono $i$ punti fondamentali (n. $\left.{ }^{\circ} 52\right)$; in qualsivoglia altro sistema lineare di genere zero e di dimensione $c^{\prime}>1$ vi sono tante curve fondamentali quanto è il numero dei punti fondamentali diminuito dell'unità.

\section{Genere $p=1$.}

XXVIII. - Qualsivoglia sistema lineare generale di genere uno è riducibile mediante trasformazioni quadratiche a un sistema di cubiche aventi $\beta$ punti comuni $(\beta=0,1,2, \ldots)$ - oppure ad uno di quartiche aventi gli stessi due punti doppi e nessun altro punto fisso comune.

Soltanto quando $i l$ grado $\grave{k} \grave{e}=8 \grave{e}$ indeciso a priori se $i l$ sistema si ridurrà a uno di cubiche $\left[a_{1}\right]_{\mu=3}$ oppure a uno di quartiche $\left[\left[a_{1}^{2} a_{2}^{2}\right]_{\mu=1}\right.$; in ambedue $i$ casi però $i l$ suo eccesso $\varepsilon \grave{e}=1$.

Vi sono in tutto 10 famiglie di sistemi lineari generali del genere $p=1$ : nessuna è di grado $k>9$, due sono del grado $k=8$, tutte le altre di gradi differenti.

In particolare:

XXIX. - Qualunque rete di curve del genere $p=1$, la quale sia determinata da punti base dati ad arbitrio, ha il grado $k=2$ ed è riducibile a una rete di $C_{3}$ passanti per 7 punti fissi arbitrarii. Il numero dei punti base, diminuito di sette unita uguaglia il numero delle linee fondamentali.

(*) Noto teorema di CRemona sulle reti omaloidiche. 
E tenuti presenti i n. ${ }^{\text {. }} 50$ e 62 :

XXX. - Qualunque fascio di curve ellittiche, il quale non sia riducibile a uno di cubiche (fascio generale o speciale di $1^{\circ}$ tipo), si può trasformare in un fascio di curve d'ordine $3 \mathrm{~m}$, dotato di 9 punti fondamentali $m$ pli $(m>1)$ e di una cubica fondamentale passante per ciascuno di esso (fascio speciale di $2^{\circ}$ tipo).

I fasci di curve ellittiche si aggruppano in una famiglia di fasci speciali di $1^{\circ}$ tipo, il cui eccesso è $\varepsilon=9$ - e in una $\infty^{\prime}$ di famiglie di fasci speciali di $2^{\circ}$ tipo, per ciascuna delle quali l'eccesso è $\varepsilon=8$.

66. Genere $p=2$.

XXXI. - Un sistema lineare generale di genere $p=2$ e di dimensione $c^{\prime}>1$ è riducibile a uno dei seguenti di minimo ordine:

(a) Sistemi di quartiche dotate di un punto doppio comune; il loro grado $k \grave{e} ₹ 12$, la loro dimensione $c^{\prime} ₹ 11$;

(b) Sistema (unico) di quintiche aventi uno stesso punto triplo, uno stesso punto doppio e nessun altro punto fisso comune; grado $k=12$, dimensione $c^{\prime}=11$

(c) Sistemi di sestiche aventi 8 punti doppi comuni; il loro grado $k$ $\grave{e} \equiv 4$, la loro dimensione $c^{\prime} \approx 3$.

In particolare:

XXXII. - Vi sono due sole famiglie di reti del genere $p=2$ e del grado $k=3$. Esse sono riducibili rispettivamente alle seguenti reti d'ordine minimo:

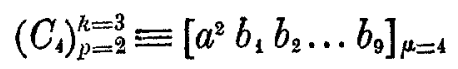

$$
\begin{aligned}
& \left(C_{6}\right)_{p=2}^{k=3} \equiv\left[\begin{array}{llll}
a_{1}^{2} a_{2}^{2} a_{3}^{2} & \ldots & a_{8}^{2} b
\end{array}\right]_{\mu=6} \text {. }
\end{aligned}
$$

In quest' ultima, due dei punti espressi dal grado $k=3$ sono variabili ed uno fisso, il quale si trova sulla cubica $\Gamma_{3} \equiv a_{1} a_{2} \ldots . a_{8} b$ determinata dai 9 punti fondamentali arbitrarii.

Onde la trasformazione di terzo grado che con questa rete si può stabilire è una degenere: ogni terna di punti conjugati del piano semplice si compone di un punto fisso e di due variabili. 


\section{$\S$ 11. Sistemi lineari generali d'ordine minimo dei generi $p=3,4$.}

67. Nelle due tabelle seguenti, relative ai generi $p=3$ e $p=4$, abbiamo segnato i sistemi generali minimi del tipo normale e i corrispondenti tipi derivati, come si sono ricavati coi metodi esposti; rimane però aperta una questione, cioè se gl'indicati gruppi di punti fondamentali definiscano tutti delle vere curve algebriche costituenti altrettanti sistemi lineari.

Quanto ai sistemi speciali di $1^{\circ}$ tipo, essi sono implicitamente contenuti, quando esistono (n. ${ }^{i}$ 54-57), nelle anzidette due tabelle. Per averli in forma esplicita bisognerebbe, per ogni singolo sistema generale di minimo ordine, esaminare se fra le $k$ intersezioni esterne ai punti fondamentali arbitrarii ve ne siano di fisse, e quante $(\S 9)$ (e riconoscere in pari tempo se alcune delle intersezioni fisse per avventura riunendosi non diano luogo a punti multipli, nel qual caso il considerato sistema generale di genere $p$ somministrerebbe in realtà un sistema speciale di genere diverso). Di questa interessante questione, che rimane pure aperta, noi non ci siamo occupati se non in casi particolari $\left({ }^{*}\right)$ nella presente Memoria, non avendo essa influenza sul problema che ne formava l'oggetto principale: quello cioè di assegnare i sistemi lineari generali di minimo ordine relativi a un dato genere $p$.

Senza pregiudicare le questioni accessorie accennate, si può tuttaria affermare che le Tabelle $\mathrm{V}$ e VI, salve le fatte riserve (xxxur e xxxv), non potranno arricchirsi di nuovi sistemi generali d'ordine minimo $\left({ }^{*}\right)$, ma ch'esse potrebbero bensì eventualmente perderne qualcuno. Uguale osservazione vale anche pei valori di $p$ qui non considerati; cosicchè col sussidio di ricerche ausiliarie, speciali pei singoli casi, i metodi esposti somministrano per ogni dato genere $p$ la totalità $\left({ }^{* *}\right)$ dei rispettivi sistemi lineari generali.

${ }^{*}$ ) Questi casi particolari provengono tutti da un medesimo sistema minimo del tipo trinomio $\left(\mathrm{n}^{\circ}{ }^{\circ} 48\right.$, Tabella III, $\left.d\right)$ cioè dal sistema generale:

$$
\left(C_{3 p}\right)_{p=p} \equiv\left[\begin{array}{lll}
a_{1}^{p} & a_{2}^{p}, \ldots a_{3}^{p} L^{p-1}
\end{array}\right]_{C_{0} 0=p}^{k_{0}=2 p-1} .
$$

Uno $b^{\prime}$ dei $2 p-1$ punti del gruppo $\left(k_{0}\right)$ è evidentemente fisso e situato sulla cubica $\Gamma_{3}$ determiuata dai 9 punti fondamentali arbitrarii; onde il detto sistema generale contiene il sistema speciale di $10^{\circ}$ tipo d'ordine minimo:

$$
\left(C_{3 p}\right)_{p=p} \equiv\left[a_{1}^{p} a_{2}^{p} \ldots a_{8}^{p} z^{p-1} b^{1}\right]_{c^{\prime}=p}^{k=2(p-1)},
$$

avente per linea fondamentale minima la cubica $\Gamma_{3} \equiv a_{1} a_{2} \ldots a_{8} b b^{\prime}$ (se $p>1$ ) e il cui grado $k$ esprime esclusivamente punti variabili (se $p>1$ ).

(**) Si tenga presente che in questo lavoro si considerano soltanto sistemi lineari dotati di singolarità ordinarie nei punti fondamentali. 
di genere qualunque.

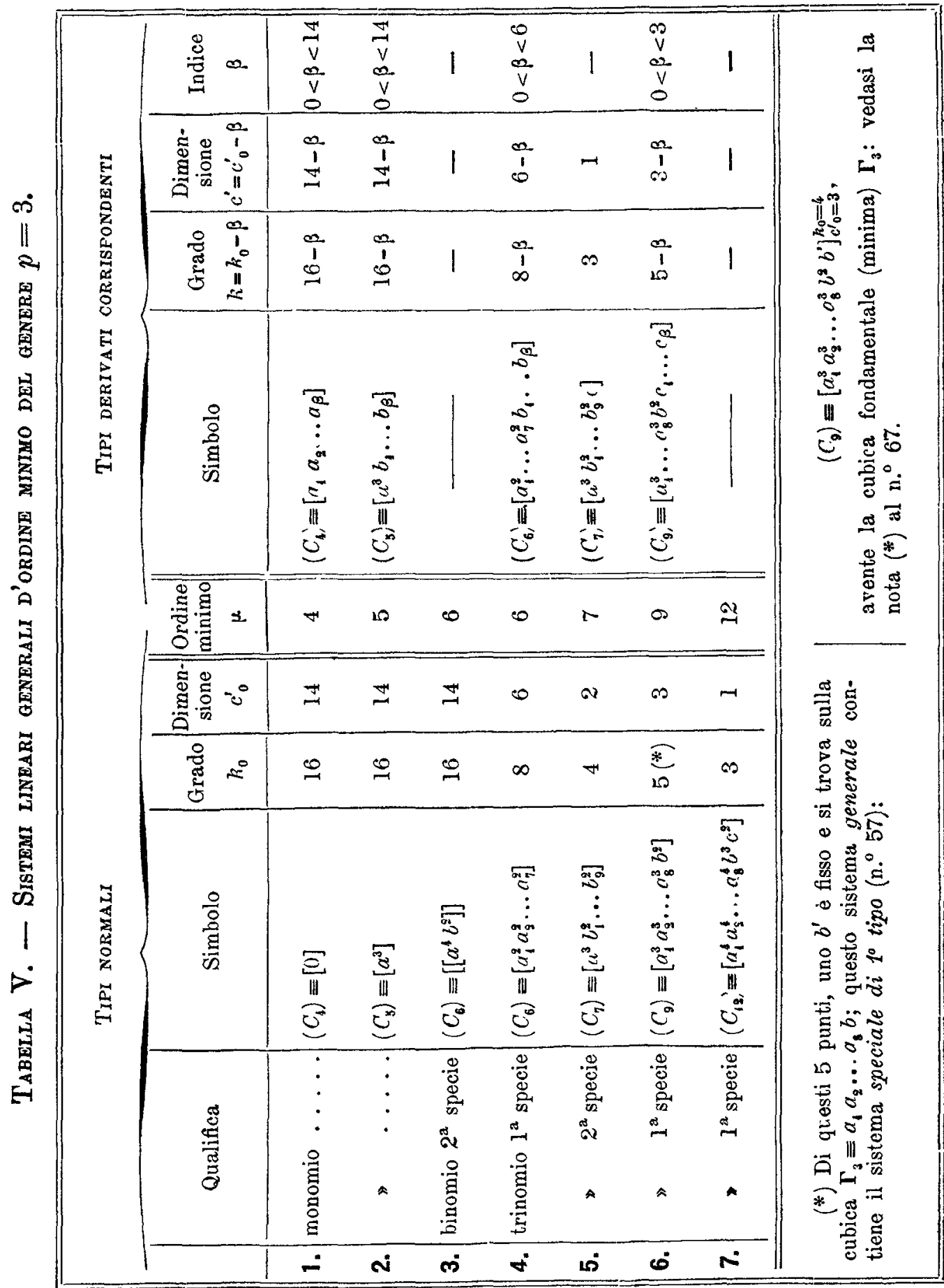

Annali di Matematica, tomo XV. 
XXXIII. - Se un sistema lineare generale di genere $p=3$ non si può ridurre a uno dei sistemi tipici contenutì in questa tabella, esso avrà la dimensione $c^{\prime} \neq 6$ e sarà trasformabile in altro d'ordine minimo del tipo trinomio.

In particolare:

$\mathrm{XXXIV.} \mathrm{-} \mathrm{Se} \mathrm{una} \mathrm{rete} \mathrm{(generale} \mathrm{e} \mathrm{determinata)} \mathrm{di} \mathrm{genere} p=3$ e di grado $k=4$ non è riducibile a una delle seguenti:

$$
\begin{aligned}
& \left(C_{4}\right)_{p=3}^{k=4} \equiv\left[\begin{array}{lllll}
a_{1} & a_{2} & a_{3} & \ldots & a_{12}
\end{array}\right]_{\mu=4} \\
& \left(C_{5}\right)_{p=3}^{k=4} \equiv\left[a^{3} b_{1} b_{2} \ldots \ldots b_{12}\right]_{\mu=5} \\
& \left(C_{6}\right)_{p=3}^{k=1} \equiv\left[a_{1}^{2} a_{2}^{2} \ldots a_{7}^{2} b_{1} \ldots b_{4}\right]_{\mu=6} \\
& \left(C_{7}\right)_{p=3}^{k=4} \equiv\left[a^{3} b_{1}^{2} b_{2}^{2} \ldots \ldots b_{9}^{2}\right]_{\mu=7} \\
& \left(C_{9}\right)_{p=3}^{h=4}=\left[a_{1}^{3} a_{2}^{3} \ldots \ldots a_{8}^{3} b^{2} c\right]_{\mu=9},
\end{aligned}
$$

essa sarà trasformabile in una rete d'ordine minimo del tipo trinomio.

Mediante l'ultima rete si può stabilire una trasformazione degenere di $4^{\circ}$ grado, inquantochè tre soltanto dei punti espressi dal grado $k=4$ sono variabili, mentre uno, situato sulla cubica $\Gamma_{3} \equiv a_{1} a_{2} \ldots a_{8} b$, rimane fisso. 
di genere qualunque.

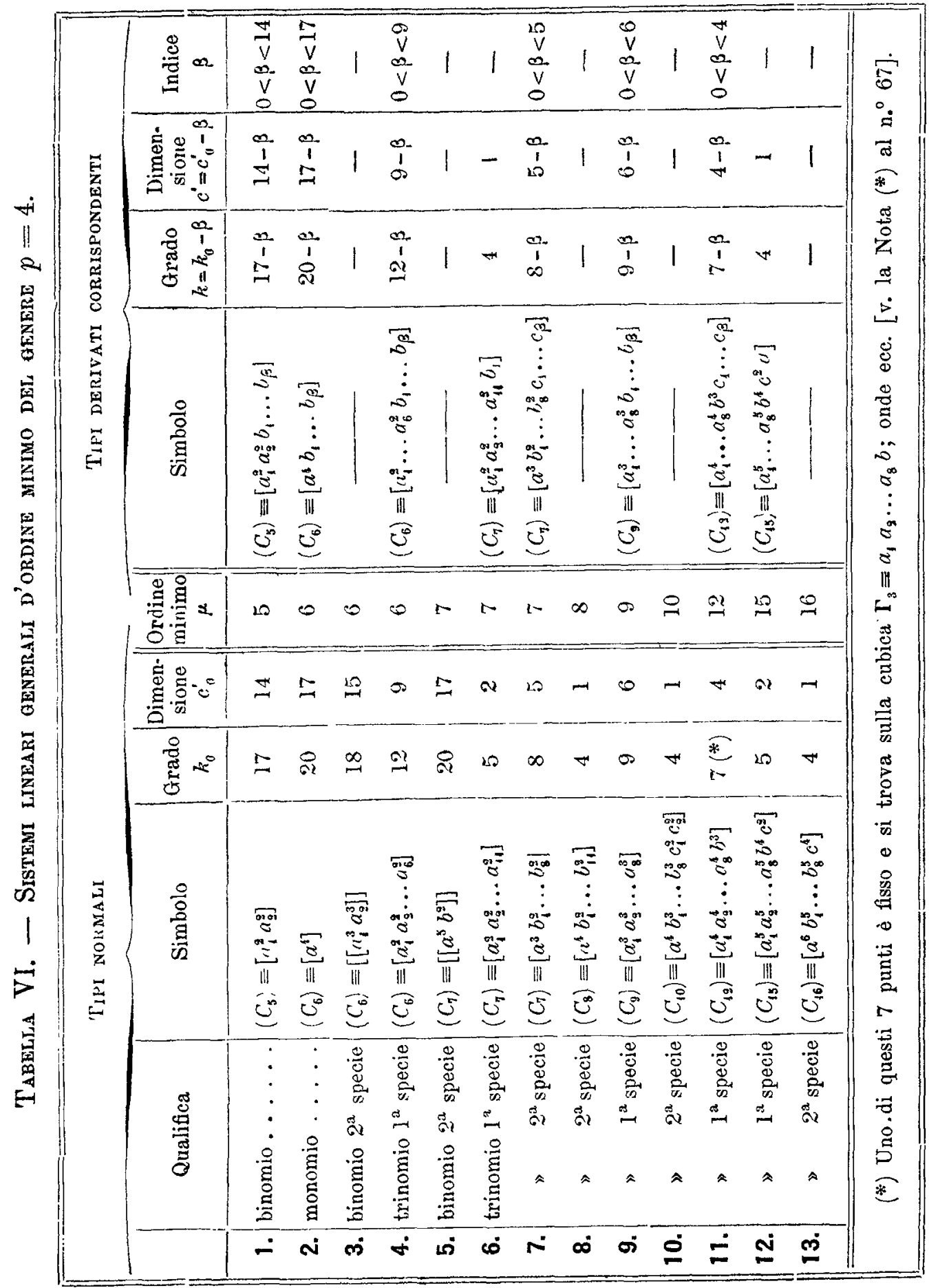


312 Jung: Ricerche sui sistemi lineari di curve algebriche, ecc.

$\mathrm{XXXY}$. - Se un sistema lineare generale di genere $p=4$ non è riducibile a uno dei sistemi tipici segnati in questa tabella, esso avrà la dimensione $c^{\prime} \rightleftharpoons 9$ e si potrà trasformare in altro d'ordine minimo del tipo trinomio.

In particolare:

XXXVI. - Una rete (generale e determinata) di genere $p=4$ e di grado $k=5$ o è riducibile a una delle seguenti:

$$
\begin{aligned}
& \left(C_{5}\right)_{p=4}^{k=5} \equiv\left[a_{1}^{2} a_{2}^{2} b_{1} \ldots \ldots b_{12}\right]_{\mu=5} \\
& \left(C_{6}\right)_{p=4}^{k=5} \equiv\left[a^{4} b_{1} b_{2} \ldots \ldots b_{15}\right]_{\mu=6} \\
& \left.\left(C_{6}\right)_{p=\downarrow}^{k=\check{z}} \equiv a_{1}^{2} a_{2}^{2} \ldots a_{6}^{2} b_{1} \ldots b_{7}\right]_{\mu=6} \\
& \left(C_{7}\right)_{k=4}^{k=3} \equiv\left[a_{1}^{2} a_{2}^{2} a_{3}^{2} \ldots \ldots a_{11}^{2}\right]_{\mu=7} \\
& \left(C_{7}\right)_{\mu=4}^{k=5} \equiv\left[a^{3} b_{1}^{2} \ldots . b_{8}^{2} c_{1} c_{2} c_{3}\right]_{\mu=7}
\end{aligned}
$$

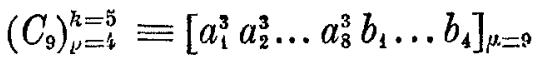

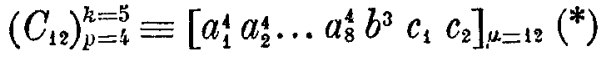

$$
\begin{aligned}
& \left(C_{15}\right)_{p=4}^{k=5} \equiv\left[a_{1}^{5} a_{2}^{5} \ldots a_{8}^{5} b^{4} c^{2}\right]_{\mu=15} \text {, }
\end{aligned}
$$

o è trasformabile in una rete d'ordine minimo del tipo trinomio.

Milano, gennaio 1887.

(*) Un'osservazione analoga a quelle fatte per le reti $\left(C_{8}\right)_{p=2}^{k=3} \mathrm{e}\left(C_{0, p=3}^{k=4}-\right.$ vedasi XXXII e XXXIV - si potrebbe qui ripetere per questa rete. 